\title{
Enhanced Acoustic Black Hole Effect in Beams with a Modified Thickness \\ Profile and Extended Platform
}

\section{Liling Tang, and Li Cheng*}

\begin{abstract}
The phenomenon of Acoustics Black Hole $(\mathrm{ABH})$ benefits from the bending wave propagating properties inside a thin-walled structure with power-law thickness variation to achieve zero reflection when the structural thickness approaches zero in the ideal scenario. However, manufacturing an ideally tailored power-law profile of a structure with embedded $\mathrm{ABH}$ feature can hardly be achieved in practice. Past research showed that the inevitable truncation at the wedge tip of the structure can significantly weaken the expected $\mathrm{ABH}$ effect by creating wave reflections. On the premise of the minimum achievable truncation thickness by the current manufacturing technology, exploring ways to ensure and achieve better $\mathrm{ABH}$ effect becomes important. In this paper, we investigate this issue by using a previously developed wavelet-decomposed semi-analytical model on an Euler-Bernoulli beam with a modified power-law profile and an extended platform of constant thickness. Through comparisons with the conventional $\mathrm{ABH}$ profile in terms of system loss factor and energy distribution, numerical results show that the modified thickness profile brings about a systematic increase in the $\mathrm{ABH}$ effect at mid-to-high frequencies, especially when the truncation thickness is small and the
\end{abstract}


profile parameter $m$ is large. The use of an extended platform further increases the $\mathrm{ABH}$ effect to broader the frequency band whilst providing rooms for catering particular low frequency applications.

Keywords: Acoustic black hole; Mexican hat wavelet-decomposed; Flexural vibration; modified thickness profile; extended platform.

\section{Introduction}

Suppression of structural vibration while maintaining its light-weight is important for various industrial applications. The Acoustics Black Holes $(\mathrm{ABH})$ effect results from the manipulation of bending wave propagation inside a thin-walled structure through thickness changes. With a tailored power-law thickness variation, the phase velocity of the bending wave gradually reduces to zero in the ideal scenario, resulting in zero wave reflection and high energy concentration at the wedge tip [1-3]. ABH effect attracts increasing attention as a promising passive vibration control method because vibration energy can be channeled and only a very small amount of damping materials is required at the energy focalization region to achieve efficient damping to flexural waves [4-9]. It also shows appealing potential in sound radiation control $[10,11]$ and energy harvesting $[12,13]$ due to the high energy concentration within a confined area.

Krylov et al [3] showed that the inevitable truncation (the residual thickness at the wedge tip) resulting from the manufacturing difficulty would significantly compromise the ideal $\mathrm{ABH}$ effect by generating wave reflections. To maximize the $\mathrm{ABH}$ effect, however, the ultimate pursuit of extremely thin wedge tip is of high cost and harsh demand for the precision machining and would also lead to tip damage of tearing and irregularities. Although Bowyer et al [14] experimentally showed that the damage on the wedge tip does not notably affect the ABH effect; 
Denis et al [15] reported that the imperfect wedge tip would reduce the reflection because of the resultant scattering effects; structures with ultra-thin or damaged tips however can hardly be applied in industry due to the structural strength problems. Therefore, on the premise of the minimum achievable truncation thickness by currently available manufacturing technology, ways maximize the ABH effect need to be explored.

Motivated by this, Bayod [16] proposed a modified thin wedge with extended constant thickness to achieve better vibration damping compared with conventional wedge. Experiments and FEM analyses were carried out to confirm this concept. Probably due to the lack of simulation tools, however, no deep explanation and parametric studies were provided in that work to guide the design of the modified wedge. Meanwhile various modified wedge thickness profiles were also proposed [13, 17]. Although similar ABH effect as the conventional profile was observed, the effect of various parameters defining the modified profiles still needs to be systematically analyzed and quantified. On the other hand, nearly all the references mentioned above focused on the ABH effect at relatively higher frequencies. Possible extension of the $\mathrm{ABH}$ effect to lower frequencies is still a great challenge and is of particular importance for applications in energy harvesting and noise control. It is understandable the none of the above could be done without a reliable and flexible simulation tool.

In this paper, we focus on seeking ways to achieve better $\mathrm{ABH}$ effect on the premise of the minimum achievable truncation thickness and the possibility of applying $\mathrm{ABH}$ effect at low frequencies. Firstly, an Euler-Bernoulli beam, with modified thickness profile, $h(x)=\varepsilon\left(x-x_{0}\right)^{m}+h_{0}$ and an extended platform, is studied using a previously developed wavelet-decomposed model $[18,19]$. Then, the effect of the additional thickness $h_{0}$ and the 
extended platform is systematically discussed through numerical simulations. Particularly, we investigate the effect of the profile parameters on the average system loss factor for different additional thicknesses and lengths of extended platform. A particular focus is also put on exploring the beneficial effect of the extended platform in the low frequency range. Finally, conclusions are drawn.

\section{Modelling of a Beam with a Modified Thickness Profile and Extended Platform}

We consider an Euler-Bernoulli beam composed of a uniform portion with a constant thickness $h_{b}$ from $x_{b 3}$ to $x_{b 4}$, and an $\mathrm{ABH}$ portion with a modified thickness profile, $h(x)=\varepsilon\left(x-x_{0}\right)^{m}+h_{0}$, from $x_{b 2}$ to $x_{b 3}$ (Fig. 1). When $x_{0}$ and $h_{0}$ are both equal to zero, it retreats to the conventional power-law thickness profile, i.e. $h(x)=\varepsilon x^{m}$. A platform of uniform thickness $h\left(x_{b 2}\right)$ is extended from the truncation point $x_{b 2}$ to point $x_{b 1}$. The beam is excited by a point force $f(t)$ at $x_{f}$ and is covered by two damping layers with variable thickness $h_{d}(x)$ from $x_{d 1}$ and $x_{d 2}$. The whole system is symmetrical with respect the mid-line of the beam. The extended platform end of the beam is free and the other end is elastically supported by artificial translational and rotational springs $[20,21]$, the stiffness of which can be adjusted to achieve various boundary conditions. The damping of both the beam and the damping layer are taken into account through complex stiffness $E$, i.e., $E=E(1+i \eta)$, where $\eta$ is the damping loss factor, assigned differently to the beam and the damping layer. A previously developed wavelet-decomposed mothed based on Lagrange's equation is used to obtain the vibration response $[18,19]$. 


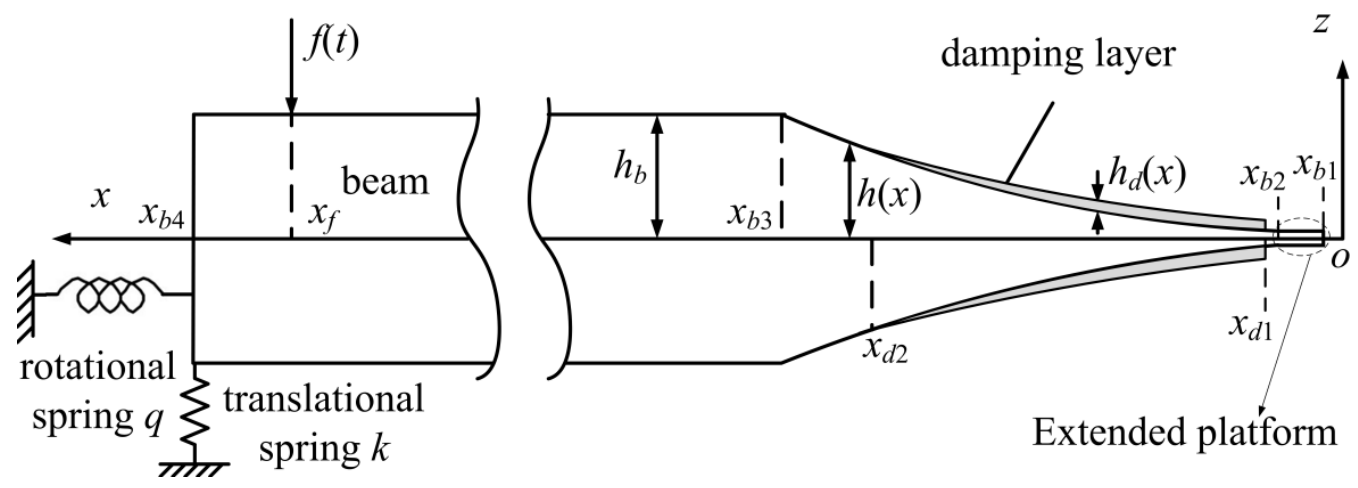

Fig. 1 An Euler-Bernoulli beam with symmetrical modified power-law profile and extended platform.

For the benefit of readers, the modeling principle is briefly recalled. The displacement field of the beam based on Euler-Bernoulli beam theory is expressed as

$$
\{u, w\}=\left\{-z \frac{\partial w}{\partial x}, w(x, t)\right\}
$$

where the vector $\{u, w\}$ represents the displacement of a point either on the beam or on the damping layers based on the assumption of perfect bonding between them. The transverse displacement $w$ can be expanded as

$$
w(x, t)=\sum_{i} a_{i}(t) \varphi_{i}(x)
$$

where $\varphi_{i}(x)$ are the assumed admissible functions and $a_{i}(t)$ the complex unknowns to be determined. As demonstrated in Ref. [18], the Mexican hat wavelets (MHW) [22, 23] are particularly suitable, compared with power series, to characterize the present rapid wavelength fluctuation along the beam, owing to the appealing properties of the wavelets in terms of scaling 
and translation. Choosing $\mathrm{MHW}\left(\varphi_{j, k}(x)\right)$ as the admissible functions, Eq. (2) can then be represented as

$$
w(x, t)=\sum_{j=0}^{m} \sum_{k} a_{j, k}(t) \varphi_{j, k}(x)
$$

where

$$
\varphi_{j, k}(x)=\frac{2}{\sqrt{3}} \pi^{-\frac{1}{4}} 2^{j / 2}\left[1-\left(2^{j} x-k\right)^{2}\right] \mathrm{e}^{-\frac{\left(2^{j} x-k\right)^{2}}{2}}
$$

$j$ is the scaling parameter (integer) to stretch or squeeze the MHW and $k$ the translation parameter (integer) to move the MHW along $x$ axis.

The extremalization of Hamiltonian function leads to the following Lagrange's equations

$$
\frac{\mathrm{d}}{\mathrm{d} t}\left(\frac{\partial L}{\partial \dot{a}_{j, k}(t)}\right)-\frac{\partial L}{\partial a_{j, k}(t)}=0
$$

where the Lagrangian of the system $L$ writes

$$
L=E_{k}-E_{p}+W
$$

in which $E_{k}$ denotes the kinetic energy of the system; $E_{p}$ the potential energy and $W$ the work done by the excited force. They can be expressed, respectively, as

$$
\begin{gathered}
E_{k}=\frac{1}{2} \int \rho\left(\frac{\partial w}{\partial t}\right)^{2} \mathrm{~d} V \\
E_{p}=\frac{1}{2} \int E I(x)\left(\frac{\partial^{2} w}{\partial x^{2}}\right)^{2} \mathrm{~d} x+\frac{1}{2} k w\left(x_{b 4}, t\right)^{2}+\frac{1}{2} q\left(\frac{\partial w\left(x_{b 4}, t\right)}{\partial x}\right)^{2} \\
W=f(t) \cdot w\left(x_{f}, t\right)
\end{gathered}
$$

Substituting Eqs. (7) to (9) into Eq. (5) yields the following linear equations

$$
\mathbf{M a ̈}(t)+\mathbf{K a}(t)=\mathbf{f}(t)
$$


where $\mathbf{M}$ and $\mathbf{K}$ are, respectively, the mass matrix and stiffness matrix; $\mathbf{a}(\mathrm{t})$ and $\mathbf{f}(\mathrm{t})$ are, respectively, the vectors of the response $a_{i}(t)$ and the force, which can be expressed as $\mathbf{a}(t)=\mathbf{A} e^{j \omega t}$ and $\mathbf{f}(t)=\mathbf{F} e^{j \omega t}$ in harmonic regime. Then Eq. (10) can be rewritten as

$$
\left[\mathbf{K}-\omega^{2} \mathbf{M}\right] \mathbf{A}=\mathbf{F}
$$

The forced vibration response can be directly obtained by solving the Eq. (11), detailed expressions of which are given in Appendix. For free vibration, setting the force vector in Eq. (11) to zero leads to the following eigenvalue equation

$$
\mathbf{M}^{-1} \mathbf{K A}=\omega^{2} \mathbf{A}
$$

which yields the natural frequencies and the corresponding mode shapes. Since the system is complex, the eigenvalues take complex forms as

$$
\omega^{2}=\omega_{n}^{2}(1+i \eta)
$$

where $\omega_{n}$ is the natural frequency and $\eta$ the corresponding modal loss factor of the system, which is an important measure of the $\mathrm{ABH}$ effect in providing effective damping to the overall system.

\section{Numerical Results and Discussions}

Consider two clamped-free beams with the same uniform portion under a harmonic driving force of $1 \mathrm{~N}$ applied at the point, $3 \mathrm{~cm}$ away from the clamped end (as shown in Fig.1). Keep the same truncation thickness $h\left(x_{b 2}\right)$ and profile parameters $\varepsilon$ and $m$, the responses of the two beams are first analyzed and compared. Case 1 involves a conventional $\mathrm{ABH}$ thickness profile $\left(h_{1}(x)=\varepsilon x^{m}\right)$ and case 2 a modified thickness profile $\left(h_{2}(x)=\varepsilon\left(x-x_{0}\right)^{m}+h_{0}\right)$. The material and geometrical parameters are listed in Table 1 . Note that truncated at $x_{b 2}=4 \mathrm{~cm}$, the prescribed truncation thickness $h\left(x_{b 2}\right), 0.02 \mathrm{~cm}$, is the same in both cases. As a benchmark system, an 
entirely uniform beam with the same thickness and length as case 1 is also given as a reference in the following analyses.

It should be noted that, in order to keep the same truncated thickness and the same uniform beam thickness, the effective length of the $\mathrm{ABH}$ part is different in cases 1 and 2 . However, we start from the assumption that the $\mathrm{ABH}$ structure is usually used as an add-on part to the existing structure (which is the uniform beam in this particular case). In this context, we would accept a slightly different ABH length as long as the results remain comparable. In the following analyses, within the range we used for $\varepsilon, m$ and other parameters, the difference in the $\mathrm{ABH}$ length is not very large, especially for cases of our interest when $m$ is large and truncation thickness is small. Meanwhile, we make sure that the cases shown in each figure are comparable by ensuring that the same damping layers are applied in all cases, starting from the free end with exactly the same thickness and length. The latter is taken as the shortest $\mathrm{ABH}$ length if different $\mathrm{ABH}$ profiles are involved in figures where comparisons are made.

\section{Table 1}

Material and geometrical parameters.

\begin{tabular}{cc}
\hline Material parameters & Geometrical parameters \\
\hline Beam & $\varepsilon=0.00125$ \\
$E_{b}=210 \mathrm{GPa}$ & $m=2$ \\
$\rho_{b}=7800 \mathrm{~kg} / \mathrm{m}^{3}$ & $x_{0}=4$ \\
$\eta_{b}=0.001$ & $h_{0}=0.02 \mathrm{~cm}$ \\
Damping layers & $h_{b}=0.32 \mathrm{~cm}$ \\
$E_{d}=5 \mathrm{GPa}$ & $h\left(x_{b 2}\right)=0.02 \mathrm{~cm}$ \\
$\rho_{d}=950 \mathrm{~kg} / \mathrm{m}^{3}$ & $l_{\mathrm{Uni}}=x_{b 4}-x_{b 3}=12 \mathrm{~cm}$ \\
$\eta_{d}=0.1$ & \\
\hline
\end{tabular}




\subsection{Effect of the additional thickness $h_{0}$}

Since tip truncation is inevitable, we will first investigate the possibility of changing the thickness profile, aiming at achieving better ABH effect than the conventional ABH profile with the same truncation. Keeping the same truncation tips with the same minimum achievable thickness $h\left(x_{b 2}\right)$, the modified thickness profile (case 2) is different from the conventional $\mathrm{ABH}$ thickness profile (case 1) in that it possesses an additional thickness term $h_{0}$. We first investigate the effect of this additional thickness term on the $\mathrm{ABH}$ effect without the consideration of the extended platform.

Figure 2 shows the mean quadratic velocity of the uniform beam portion and the energy ratio $\Gamma\left(\Gamma=10 \log \frac{\left\langle V^{2}\right\rangle_{\mathrm{ABH}}}{\left\langle V^{2}\right\rangle_{\text {Unif }}}\right)$ between the $\mathrm{ABH}$ portion and the uniform portion for three different beams without damping layers. It can be seen that the overall vibration level of the uniform portion of both beams with the conventional and modified $\mathrm{ABH}$ profiles are slightly lower at higher frequencies compared with their uniform counterpart as a result of $\mathrm{ABH}$ effect. However, due to the existence of the truncation, flexural waves are reflected back in the absence of the damping layers, which explains the barely noticeable reduction in the vibration level. Meanwhile, the modified thickness profile takes better ABH effect than the conventional profile by slightly reducing the vibration level at higher frequencies as shown in Fig. 2 (a). This can be further confirmed in the Fig. 2(b) which shows a clear vibration energy shift to the ABH portion, in case 2 more than in case 1 . 
(a)

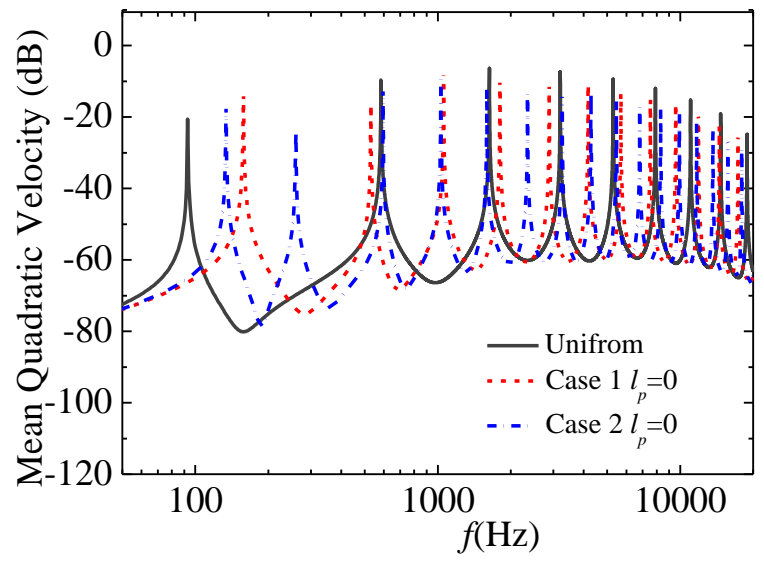

(b)

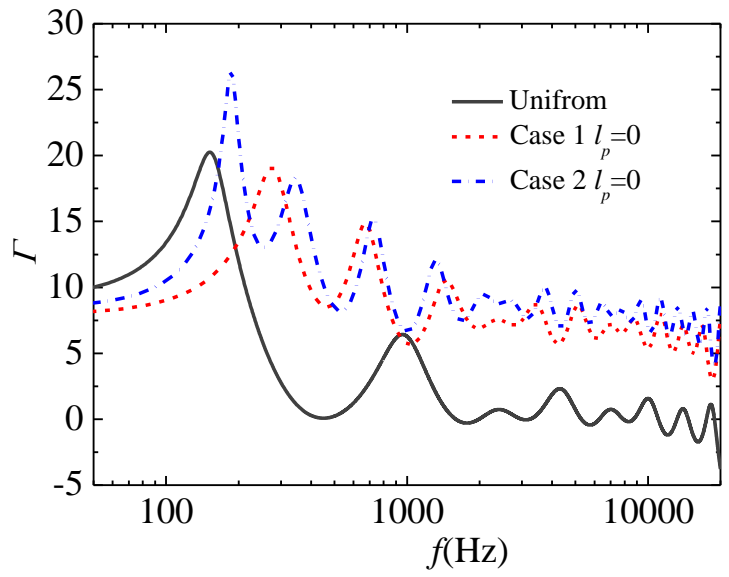

Fig. 2. (a) The mean quadratic velocity of the uniform beam portion, and (b) the ratio of the mean quadratic velocity of the $\mathrm{ABH}$ portion to the uniform beam portion for three different beam cases without damping layers.

Since the damping layers take the upmost effect near the truncation tip [18]. Fig. 3 compares the system loss factors for the same three beam cases when same length of damping layers applied. Compared with the entirely uniform beam, while the system loss factor in case 1 with conventional $\mathrm{ABH}$ profile being significantly increased at higher frequencies due to the ABH effect, that of case 2 with the modified profile is nearly doubled. Meanwhile, different from case 1, the lower-order modal loss factor in case 2 also attains noticeable increase. Thus, the overall reduction in the vibration level of the uniform portion in case 2 is also larger than that in case 1, in comparison with the entirely uniform beam as revealed in Fig. 4, reaching a level as high as $19.2 \mathrm{~dB}$. 


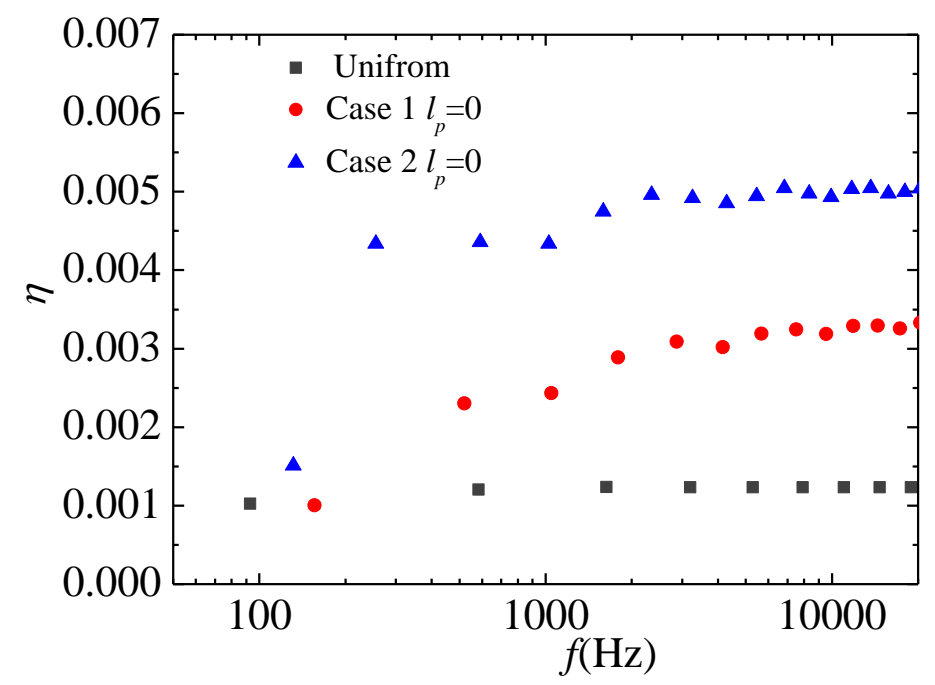

Fig. 3 The system loss factors for three different beam cases with same length of damping layers.

(a)

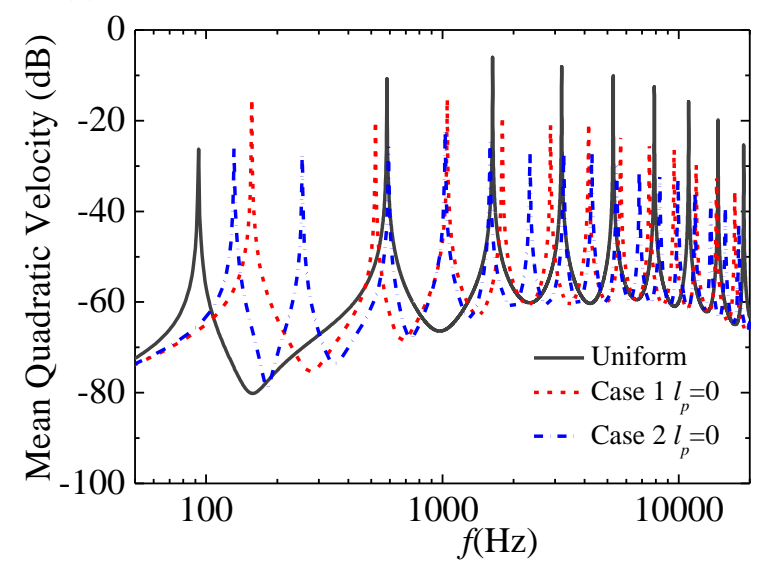

(b)

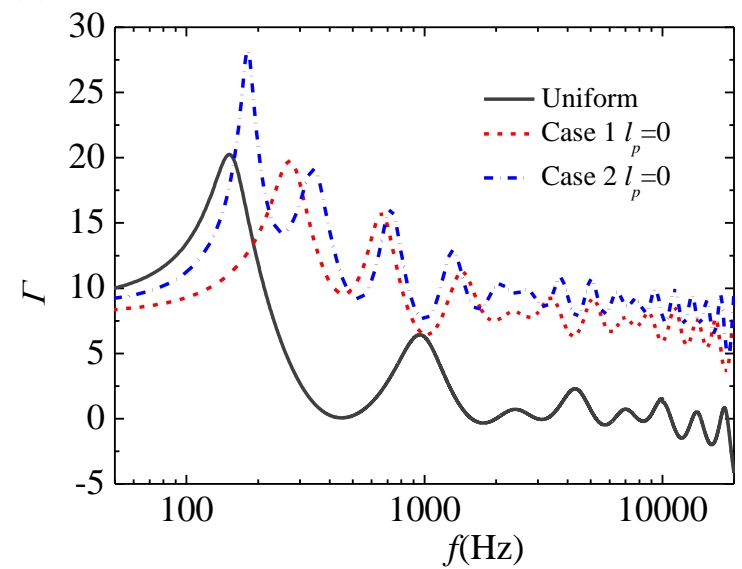

Fig. 4 (a) The mean quadratic velocity of the uniform beam portion, and (b) the ratio of the mean quadratic velocity of the $\mathrm{ABH}$ portion to the uniform beam portion for three different beam cases with same length of damping layers.

Two plausible reasons could explain the reason why the modified thickness profile with an additional thickness $h_{0}$ outperforms the conventional ABH profile for the same given thickness truncation. The mode shapes of the entire beam for the two cases are shown and compared in Fig. 
5. It can be seen that case 2 with modified profile promotes larger structural deformation at the ABH portion for both the first and one representative higher-order (tenth) mode shape. This enables more energy concentration on the $\mathrm{ABH}$ portion, conducive to energy absorption by the damping layers. From the perspective of the geometrical acoustic theory, the total wave reflection coefficient $R_{0}$ can be expressed as $R_{0}=\exp \left(-2 \int_{x_{0}}^{x} \operatorname{Im} k(x) \mathrm{d} x\right)$ [3], in which the local wavenumber $k(x)=12^{1 / 4}\left(\omega / c_{l}\right)^{1 / 2} h(x)^{-1 / 2}$ with $c_{l}=\sqrt{E_{b}\left(1+i \eta_{b}\right) / \rho_{b}}$ being the velocity of longitudinal waves. Therefore, $R_{0}$ is negatively correlated with the integration term $\int_{x_{0}}^{x} h(x)^{-1 / 2}$. The derivatives of the thickness profiles of case 1 and case 2 are respectively $h_{1}^{\prime}(x)=\varepsilon m x^{m-1}$ and $h_{2}^{\prime}(x)=\varepsilon m\left(x-x_{0}\right)^{m-1}$. It can be seen that $h_{1}^{\prime}(x)$ is always larger than $h_{2}^{\prime}(x)$. Therefore, given the same starting truncation thickness $x_{b 2}$, the thickness at any point along the beam in case 1 is always larger than that in case 2, i.e. $h_{1}(x)^{-1 / 2}<h_{2}(x)^{-1 / 2}$. On the other hand, for the same beam thickness $h\left(x_{b 3}\right)$, the corresponding $x$ of case 1 is constantly smaller than that of case 2 , i.e. $x_{b 3 \_}<$ $x_{b 3 \_2 .}$ Consequently, the integration $\int_{x_{b 2}}^{x_{b 3}{ }_{2} 2} h_{2}(x)^{-1 / 2}$ is always larger than $\int_{x_{b 2}}^{x_{b 3}{ }_{1}} h_{1}(x)^{-1 / 2}$, resulting in a lower reflection coefficient in case 2 as compared to case 1. This implies better ABH effect achieved by the case 2 for any $\varepsilon$ and $m$ as observed above. 
(a)

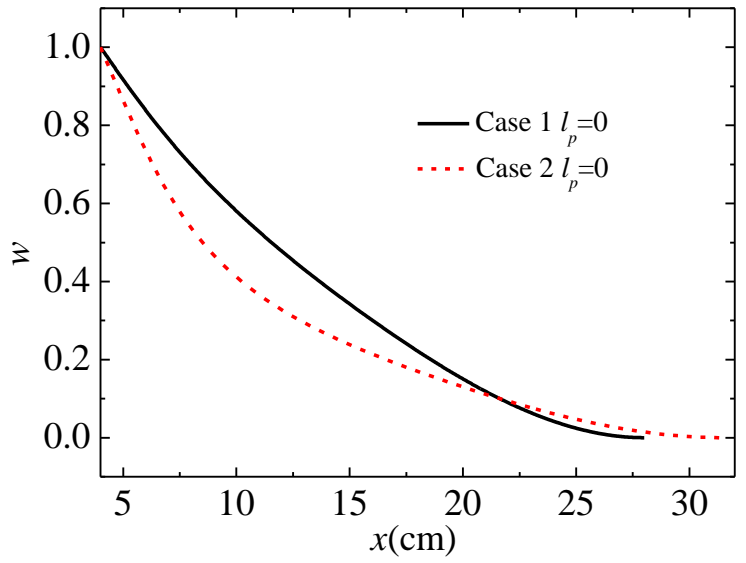

(b)

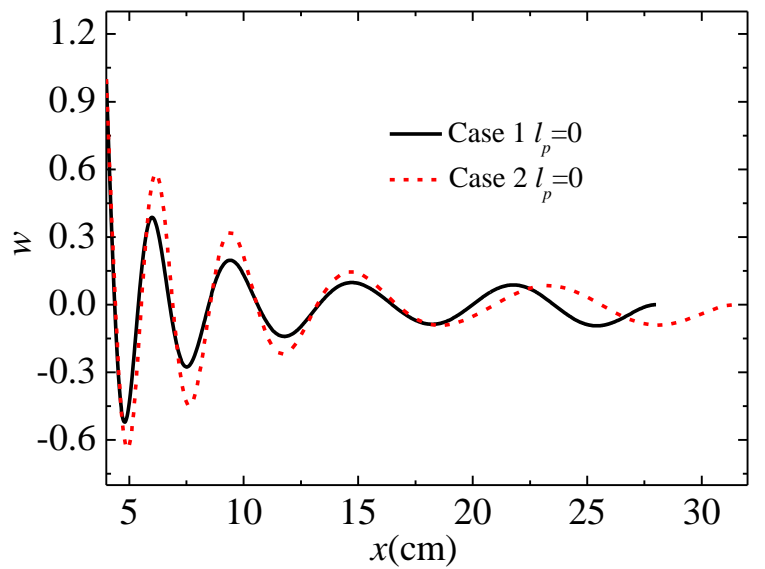

Fig. 5 Mode shape comparison for two cases: (a) first mode; (b) tenth mode.

To quantify the broadband $\mathrm{ABH}$ feature of the modified profile, the average system loss factor is used and applied to both thickness profiles cases. The band is defined starting from a cut-on frequency $f_{\text {cut-on, }}$ at which corresponding wavelength $\lambda$ of the incoming wave approaches and starts to be shorter than the characteristic $\mathrm{ABH}$ dimension, i.e. the length of the $\mathrm{ABH}$ part, so that the incoming wave can interact more effectively with the $\mathrm{ABH}$ element. For $\lambda=\frac{C_{b}}{f_{\text {cut-on }}} \leq L_{A B H}$ and $C_{b}=\sqrt[4]{\frac{E_{b} I \omega^{2}}{\rho_{b} A}}=\left[4 \pi f h_{b} \sqrt{\frac{E_{b}}{12 \rho_{b}}}\right]^{\frac{1}{2}}$, we get $f_{\text {cut }-o n}=\frac{4 \pi h_{b}}{L_{A B H}^{2}} \sqrt{\frac{E_{b}}{12 \rho_{b}}}$. Then, the increase in the average system damping loss factor is defined as $\Delta \bar{\eta}=\frac{1}{m} \sum_{p}^{p+m}\left(\eta_{2 p}+\eta_{2(p+1)}+\ldots .+\eta_{2(p+m)}\right)-\frac{1}{n} \sum_{q}^{q+n}\left(\eta_{1 q}+\eta_{1(q+1)}+\ldots .+\eta_{1(q+n)}\right)$, where the first subscript of $\eta$ denotes the case number and the second subscript denotes the mode number above the cut-on frequency.

Figure 6 shows the effect of $h_{0}$ (i.e. the truncation thickness) on $\Delta \bar{\eta}$ for different parameters (frequency band chosen as $10000 \sim 50000 \mathrm{~Hz}$ ). It can be seen that the increase in the $\mathrm{ABH}$ effect by the modified profile is much more noticeable as $h_{0}$ reduces, due to the fact that 


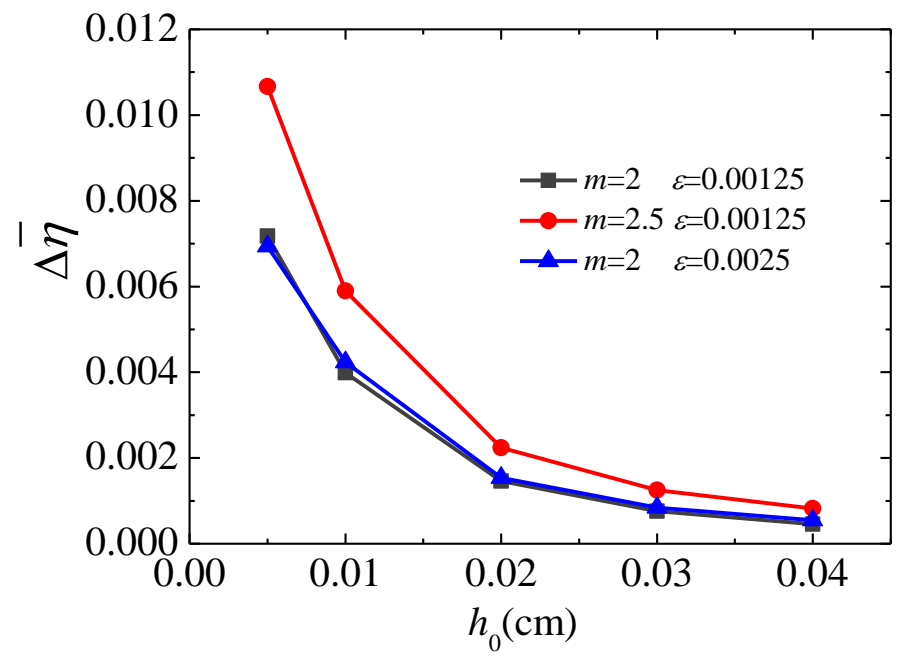

Fig. 6 Average increase in system damping loss factor of case 2 compared with case 1 under different parameters of thickness profile.

\subsection{Effect of the extended platform}

Using both ideal and modified thickness profiles, the truncated tip is extended to form a platform of constant thickness. Numerical analyses are performed to explore the possibility of 
improving $\mathrm{ABH}$ effect at both high and low frequencies using the extended platform. Fig. 7 compares the system loss factors for both cases with and without the platform when $\mathrm{ABH}$ portion (extended platforms also considered as a part of the $\mathrm{ABH}$ portion for convenience) is covered by damping layers with same length. It can be seen that the extended platform, with a length $l_{p}=4 \mathrm{~cm}$, significantly increases the system damping at higher frequencies for both thickness profiles. As defined before, the cut-on frequency $\left(f_{\text {cut-on }}=\frac{4 \pi h_{b}}{L_{A B H}^{2}} \sqrt{\frac{E_{b}}{12 \rho_{b}}}\right)$ actually shifts to lower frequencies because the characteristic dimension $L_{A B H}$ of the $\mathrm{ABH}$ region is enlarged by the extended platform, i.e. $L_{A B H}=l_{A B H}+l_{P}$. Taking case 1 as an example, the cut-on frequency without platform is roughly $2000 \mathrm{~Hz}$, while that with the platform reduces to about $1100 \mathrm{~Hz}$, as shown in Fig. 7. On the other hand, compared with the cases without platform, the system loss factor with a platform also significantly increases below the cut-on frequency. Therefore, the extended platform allows achieving better broadband $\mathrm{ABH}$ effect while providing the possibility to lower its effective region. Owing to the additional benefit of the additional thickness $h_{0}$ revealed above, the modified thickness profile further enhances the $\mathrm{ABH}$ effect compared with the conventional ABH profile, as demonstrated in Fig.7. 


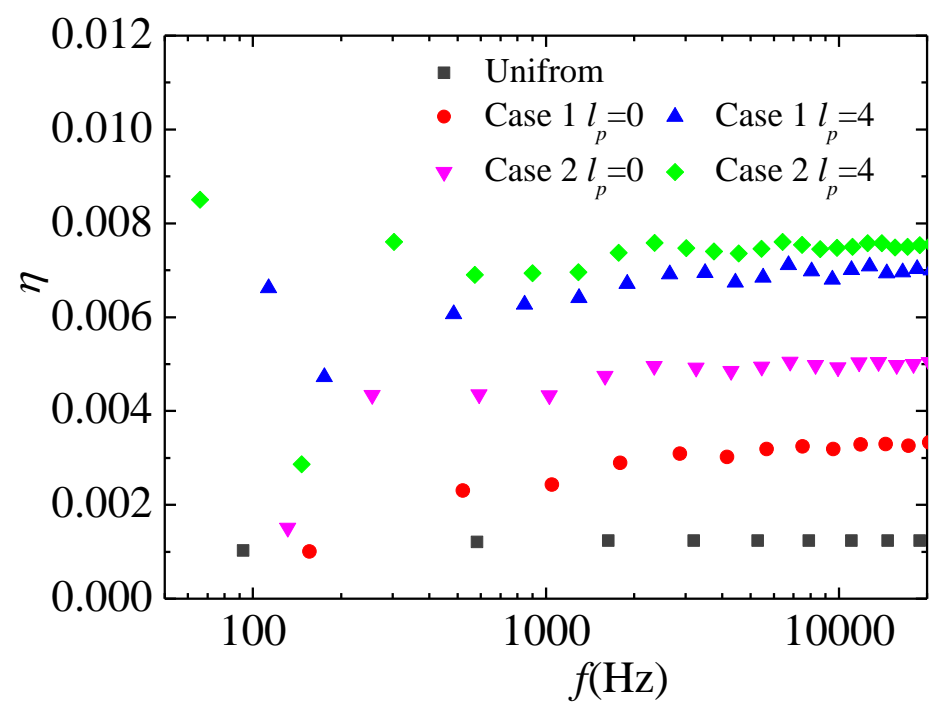

Fig. 7 System loss factors for three different beam cases with and without extended platform when damping layers with same length applied.

Focusing on case 2 with the modified thickness profile, Fig. 8 shows the vibration level of the uniform part and the energy ratio of the beams with and without platform when damping layers applied. As can be seen from Fig. 8 (a), the overall vibration level of the uniform beam portion is reduced with the use of the extended platform, which is systematic at higher frequencies, but more or less at the lower resonant frequencies. It is understandable that the lower frequency alteration in the system damping depends more on the modal characteristics of the system, which certainly deserves a closer examination. In general, however, the platform allows better energy focalization in the $\mathrm{ABH}$ part at higher frequencies (Fig. 8 (b)). Meanwhile, the first peak in the energy ratio curves increases significantly while the corresponding frequency shifts to much lower frequency than the case without platform, which implies more effective $\mathrm{ABH}$ effect at a lower frequency as well. 
(a)

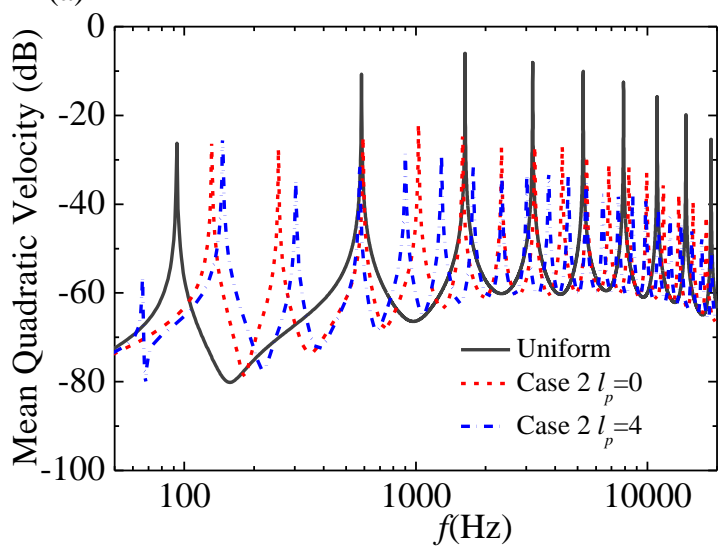

(b)

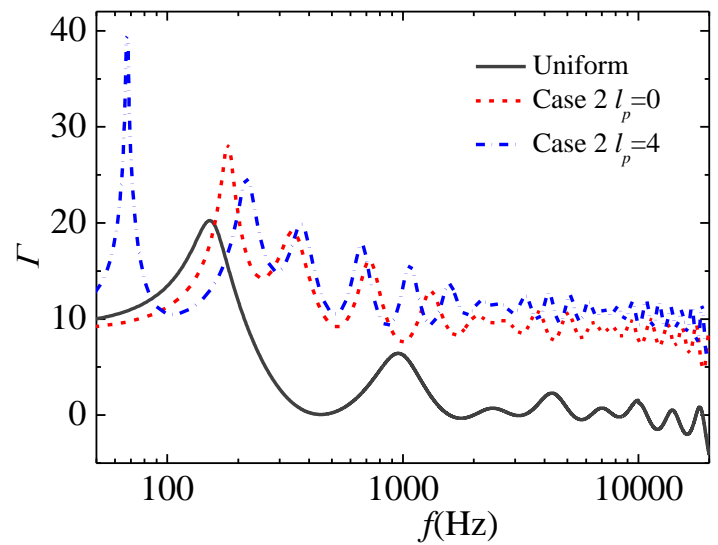

Fig. 8 (a) Mean quadratic velocity of the uniform beam portion, and (b) ratio of mean quadratic velocity of the $\mathrm{ABH}$ portion to the uniform beam portion for case 2 with and without extended platform when damping layers with same length applied.

To further explain the observed phenomena, Fig. 9 shows the mode shape with and without the extended platform. Similar to Fig. 5, the case with a platform involves more significant structural deformation at the $\mathrm{ABH}$ portion than the one without platform at the first mode, allowing better energy concentration in the $\mathrm{ABH}$ portion. For higher-order mode (tenth mode as an example), the extended platform prolongs the active area of the $\mathrm{ABH}$ by extending the intensive wave packet to the platform area after being compressed by the $\mathrm{ABH}$ profile.

Fig. 10 shows the effect of the length of the extended platform on the system loss factor. As the length increases, the system loss factor also increases in a broadband region, not necessarily proportional to the increase in the length of the platform. Below the cut-on frequency, although the damping enhancement is observable, the tendency, however, is less systematic for different lengths of the platform. Nevertheless, the observation that the first system modal loss factors increases and the effective frequency is shifted to lower frequencies with the increasing platform length still holds. Again, the phenomenon strongly depends on the modal behavior of the 
structure. Therefore, the length of the platform should be properly selected to target particular application frequency range with additional consideration of the system dimension.

(a)

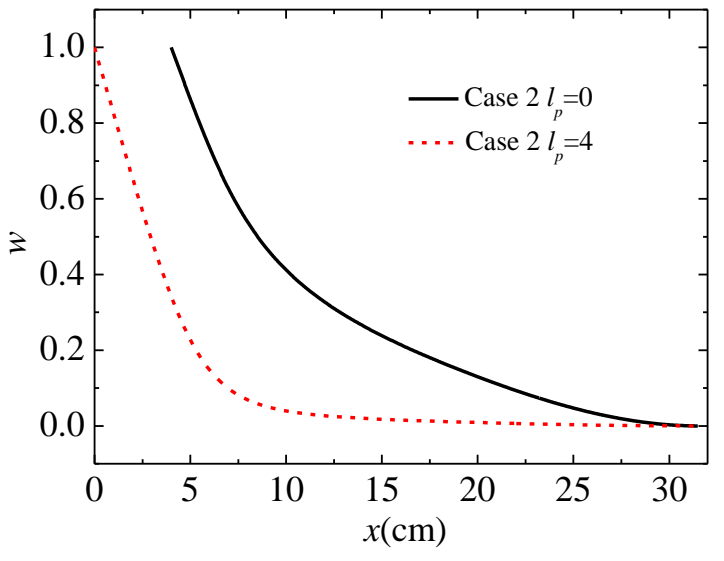

(b)

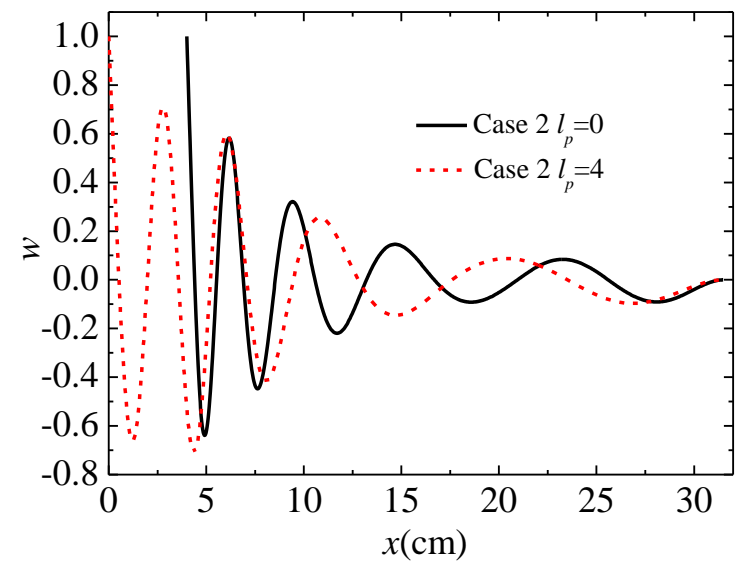

Fig. 9 Mode shape comparison for case 2 with and without extended platform: (a) first mode; (b) tenth mode.

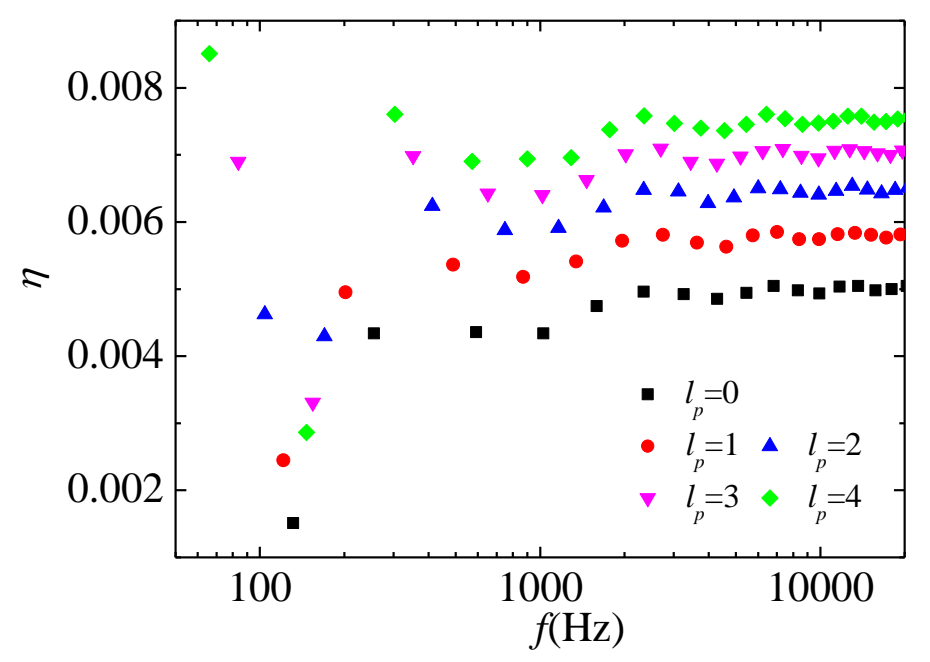

Fig. 10 System loss factor for case 2 with different lengths of the extended platform when damping layers with same length applied. 
Effect of different thickness profile parameters is revealed in Fig. 11, which shows the influences of the length of the extended platform on the average system loss factor for case 2 and the corresponding damping enhancement compared with case 1, respectively. It can be seen that, irrespective of the profile parameters, the average system loss factor systematically increases as the length of platform increases. A larger power parameter $m$ further helps enhancing the effect. The average system loss factor is lower for larger $\varepsilon$ and shorter platform, but higher when the platform becomes longer. In terms of damping enhancement in case 2 compared with case 1, $\Delta \bar{\eta}$ also increases with $m$ and $\varepsilon$. The decreasing trend of $\Delta \bar{\eta}$ with the length of the extended platform suggests that, although the damping being enhanced for both ideal and modified thickness profiles with the use of extended platform, the modified thickness profile, however, allows achieving more significant improvement as compared to the ideal thickness profile for shorter platform. This could be an additional advantage for using modified profile, since an excessively long extended platform may not be feasible in practical applications.

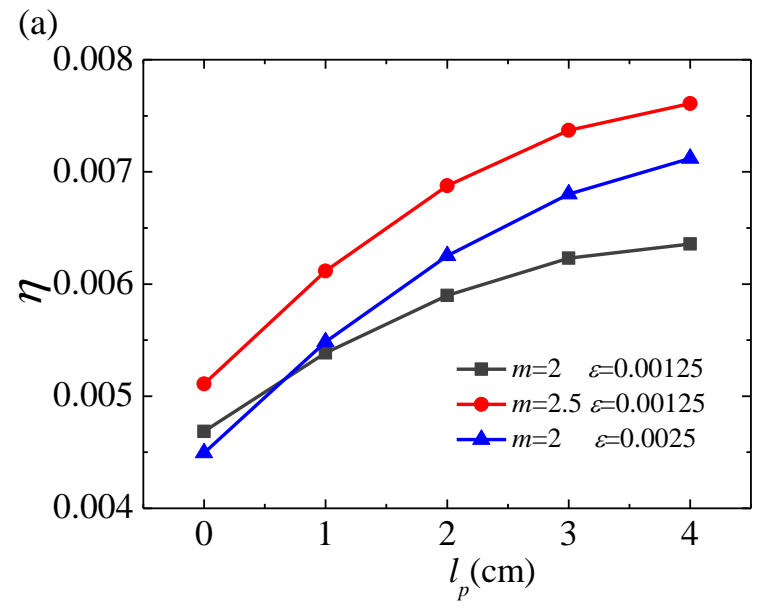

(b)

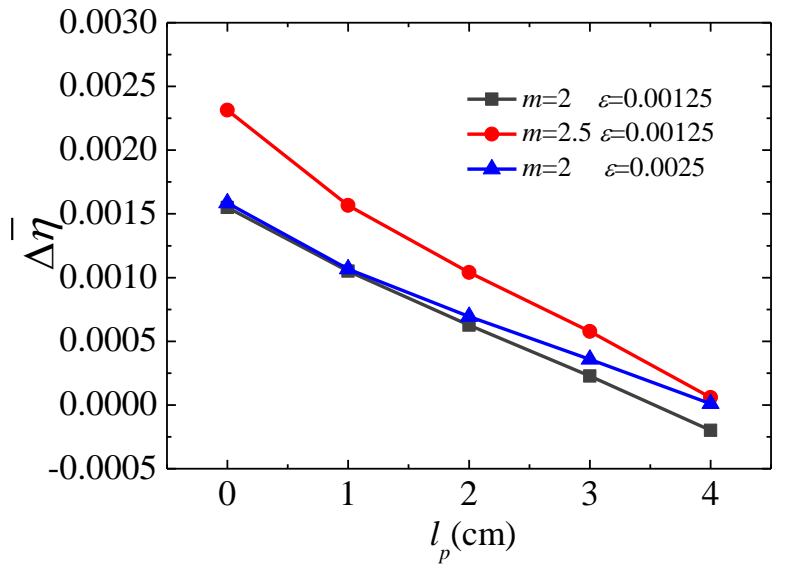

Fig. 11 (a) Average system loss factor in case 2 within broadband effective frequency range and (b) Damping increase compared with case 1 when damping layers with same length applied. 
To further examine the effect of the extended platform at lower frequencies, the first system modal loss factor $\left(\eta_{1}\right)$ and the frequency of the first peak $f_{1}$ on the energy ratio curve for different platform lengths and profile parameters are plotted in Fig. 12. For shorter platform length, $\eta_{1}$ seems to be insensitive to the profile parameters. With the increase in the platform length, however, $\eta_{1}$ rapidly increases before reaching a certain relatively stable level. Meanwhile, larger $m$ and $\varepsilon$ are beneficial. Fig. 12(b) shows that $f_{1}$ is also down-shifted more significantly for larger $m$ and $\varepsilon$, which confirms the favorable effect of the extended platform on expanding $\mathrm{ABH}$ effect further down to lower frequencies.

(a)

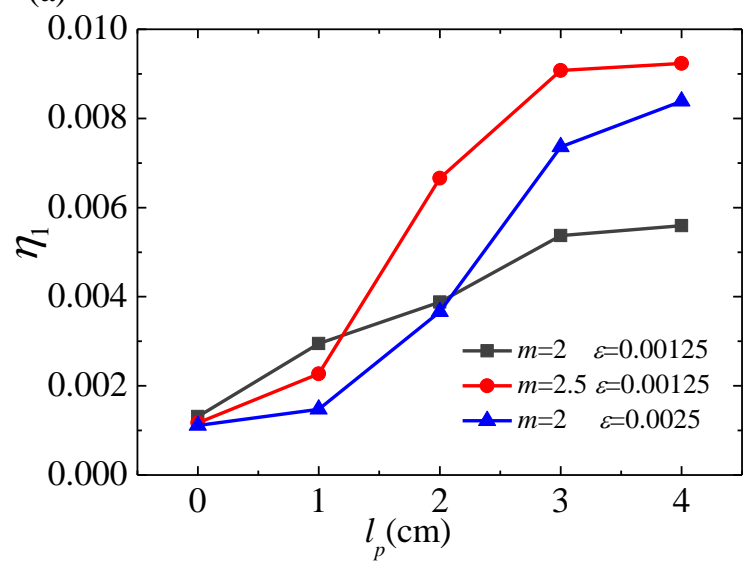

(b)

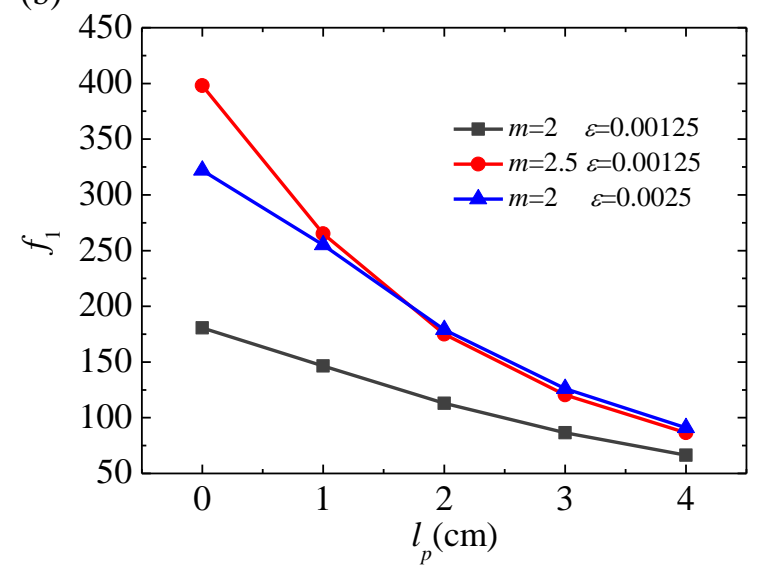

Fig. 12. (a) The first system modal loss factor and (b) The frequency of the first peak in the energy ratio curve $\Gamma$ in case 2 when damping layers with same length applied.

\section{Conclusions}

In this paper, we investigate an Euler-Bernoulli beam with a modified thickness profile and extended platform using a previously developed wavelet-decomposed semi-analytical model based on Lagrange's equation. On the premise of the same minimum achievable truncation 
thickness, the vibration level of the uniform part of the beam, the energy distribution and the system loss factor with the modified thickness profile are systemically investigated and compared with its counterpart with conventional $\mathrm{ABH}$ profile when damping layers are applied. It is shown that the ABH effect can be significantly enhanced through the use of the modified thickness profile in terms of vibration reduction of the uniform part, energy distribution and the system loss factor. The observed improvement on $\mathrm{ABH}$ effect can be explained using the geometrical acoustical theory, which indicates a reduced wave reflection coefficient as a result of the modified thickness profile irrespective of $m$ and $\varepsilon$. The improvement in the $\mathrm{ABH}$ effect is more significant with larger power parameter $m$ and smaller truncation thickness, with negligible influence of parameter $\varepsilon$.

The use of an extended platform brings about two positive effects: an enhancement of the overall system damping in a broad frequency band above the cut-on frequency; and an appreciable shift of the $\mathrm{ABH}$ effect towards low frequency. For the former, the system loss factors increases with the length of the extended platform, especially with a larger power index $m$. The effect of parameter $\varepsilon$, however, depends on the length of the extended platform. Therefore, an optimal configuration needs to be worked out in order to find the best combination among different parameters. For the latter, the extended platform can significantly enlarge the first peak of energy ratio and shift it to lower frequency, which provides the possibility of catering $\mathrm{ABH}$ effect for lower frequency applications. The tuning of this phenomenon, however, strongly depends on the modal behavior of the whole system, which again requires meticulous analyses using a simulation tool (as the one used in this work) to optimize the $\mathrm{ABH}$ performance for particular applications. 


\section{Acknowledgements}

The authors would like to thank the Research Grant Council of the Hong Kong SAR (PolyU 152009/15E and PolyU 152026/14E), National Science Foundation of China (No. 11532006) and the NUAA State Key Laboratory Program under Grant MCMS-0514K02 for financial support. 


\section{Reference}

[1] V.V. Krylov, A.L. Shuvalov, Propagation of localised flexural vibrations along plate edges described by a power law, Proceedings of the Institute of Acoustics 22 (2) (2000) 263-270.

[2] V.V. Krylov, Localised acoustic modes of a quadratically shaped solid wedge, Moscow University Physics Bulletin 45 (6) (1990) 65-69.

[3] V.V. Krylov, F. J. B. S. Tilman, Acoustic 'black holes' for flexural waves as effective vibration dampers, Journal of Sound and Vibration274 (2004) 605-619.

[4] V.V. Krylov, New type of vibration dampers utilizing the effect of acoustic black holes, Acta Acustica United with Acustica 90(5) (2004) 830-837.

[5] D.J. O’Boy, V.V. Krylov, Damping of flexural vibrations in circular plates with tapered central holes, Journal of Sound and Vibration330 (2011) 2220-2236.

[6] D.J. O’Boy, E.P. Bowyer, V.V. Krylov, Point mobility of a cylindrical plate incorporating a tapered hole of power-law profile, Journal of the Acoustical Society of America129(6) (2011) $3475-3482$.

[7] V.B. Georgiev, J. Cuenca, F. Gautier, L. Simon, V. V. Krylov, Damping of structural vibrations in beams and elliptical plates using the acoustic black hole effect, Journal of Sound and Vibration 330 (2011) 2497-2508.

[8] E.P. Bowyer, D.J. O’Boy, V.V. Krylov, Damping of flexural vibrations in plates containing ensembles of tapered indentations of power-law profile, Proceeding of Meetings on Acoustics 18(2013) 030003.

[9] V.V. Krylov, R.E.T.B. Winward, Experimental investigation of the acoustic black hole effect for flexural waves in tapered plates, Journal of Sound and Vibration 300 (2007) 43-49. 
[10] E.P. Bowyer, V.V. Krylov, Experimental study of Sound radiation by plates containing circular indentations of power-law profile, Applied Acoustics 88(2015) 30-37.

[11] S.C. Conlon, J.B. Fahnline, Numerical analysis of the vibroacoustic properties of plates with embedded grids of acoustic black holes, Journal of the Acoustical Society of America137(1) (2015) 447-457.

[12] L.X. Zhao, S.C. Conlon, F. Semperlotti, Broadband energy harvesting using acoustic black hole structural tailoring, Smart Materials and Structures 23 (2014) 065021.

[13] L.X. Zhao, S.C. Conlon, F. Semperlotti, An experimental study of vibration based on energy harvesting in dynamically tailored structures with embedded acoustic black holes, Smart Materials and Structures 24 (2015) 065039.

[14] E.P. Bowyer, D.J. O’Boy, V.V. Krylov, J.L. Horner, Effect of geometrical and material imperfections on damping flexural vibrations in plates with attached wedges of power law profile, Applied Acoustics 73 (2012) 514-523.

[15] V. Denis, A. Pelat, F. Gautier, Scattering effects induced by imperfections on an acoustic black hole placed at a structural waveguide termination, Journal of Sound and Vibration 362 (2016) 56-71.

[16] J.J. Bayod, Experimental study of vibration damping in a modified elastic damping in a modified elastic wedge of power-law profile, Journal of Vibration and Acoustics 133 (2011) 061003.

[17] V. Denis, F. Gautier, A. Pelat, J. Poittevin, Measurement and modelling of the reflection coefficient of an Acoustic Black Hole termination, Journal of Sound and Vibration 349 (2015) 67-79. 
[18] L.L. Tang, L. Cheng, H.L, Ji, J.H. Qiu, Characterization of Acoustic Black Hole effect using a one-dimensional fully-coupled and wavelet-decomposed semi-analytical model, Journal of Sound and Vibration 374 (2016) 172-184.

[19] L.L. Tang, L. Cheng, Loss of acoustic black hole effect in a structure of finite size, Applied Physics Letters 109 (2016) 014102.

[20] L. Cheng, R. Lapointe, Vibration attenuation of panel structures by optically shaped viscoelastic coating with added weight considerations, Thin-Walled Structures 21 (1995) 307-326.

[21] L. Cheng, Vibroacoustic modeling of mechanically coupled structures: artificial spring technique applied to light and heavy medium, Shock and Vibration 3(3) (1996) 193-200.

[22] I. Daubechies, Ten lectures on wavelets, CBM-NSF conference series in applied mathematics, Philadelphia: SIAM ED, 1992.

[23] T. Hou, H. Qin, Continuous and discrete Mexican hat wavelet transform on manifolds, Graphical Models 74(2012)221-232. 


\section{Figures and Tables Caption}

Fig. 1 An Euler-Bernoulli beam with symmetrical modified power-law profile and extended platform.

Fig. 2 (a) The mean quadratic velocity of the uniform beam portion, and (b) the ratio of the mean quadratic velocity of the $\mathrm{ABH}$ portion to the uniform beam portion for three different beam cases without damping layers.

Fig. 3 The system loss factors for three different beam cases with same length of damping layers.

Fig. 4 (a) The mean quadratic velocity of the uniform beam portion, and (b) the ratio of the mean quadratic velocity of the $\mathrm{ABH}$ portion to the uniform beam portion for three different beam cases with same length of damping layers.

Fig. 5 Mode shape comparison for two cases: (a) first mode; (b) tenth mode.

Fig. 6 Average increase in system damping loss factor of case 2 compared with case 1 under different parameters of thickness profile.

Fig. 7 System loss factors for three different beam cases with and without extended platform when damping layers with same length applied.

Fig. 8 (a) Mean quadratic velocity of the uniform beam portion, and (b) ratio of mean quadratic velocity of the $\mathrm{ABH}$ portion to the uniform beam portion for case 2 with and without extended platform when damping layers with same length applied.

Fig. 9 Mode shape comparison for case 2 with and without extended platform: (a) first mode; (b) tenth mode. 
Fig. 10 System loss factor for case 2 with different lengths of the extended platform when damping layers with same length applied.

Fig. 11 (a) Average system loss factor in case 2 within broadband effective frequency range and (b) Damping increase compared with case 1 when damping layers with same length applied.

Fig. 12. (a) The first system modal loss factor and (b) The frequency of the first peak in the energy ratio curve $\Gamma$ in case 2 when damping layers with same length applied.

Table 1 Material and geometrical parameters. 

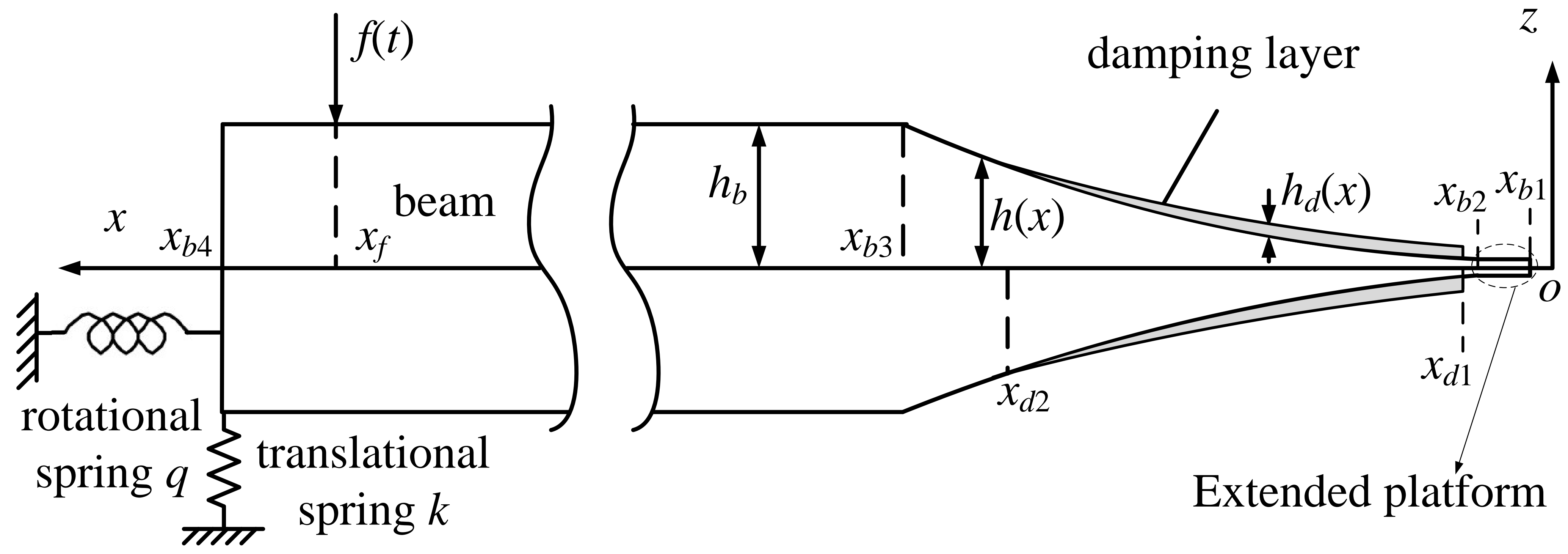
Fig. 2

(a)

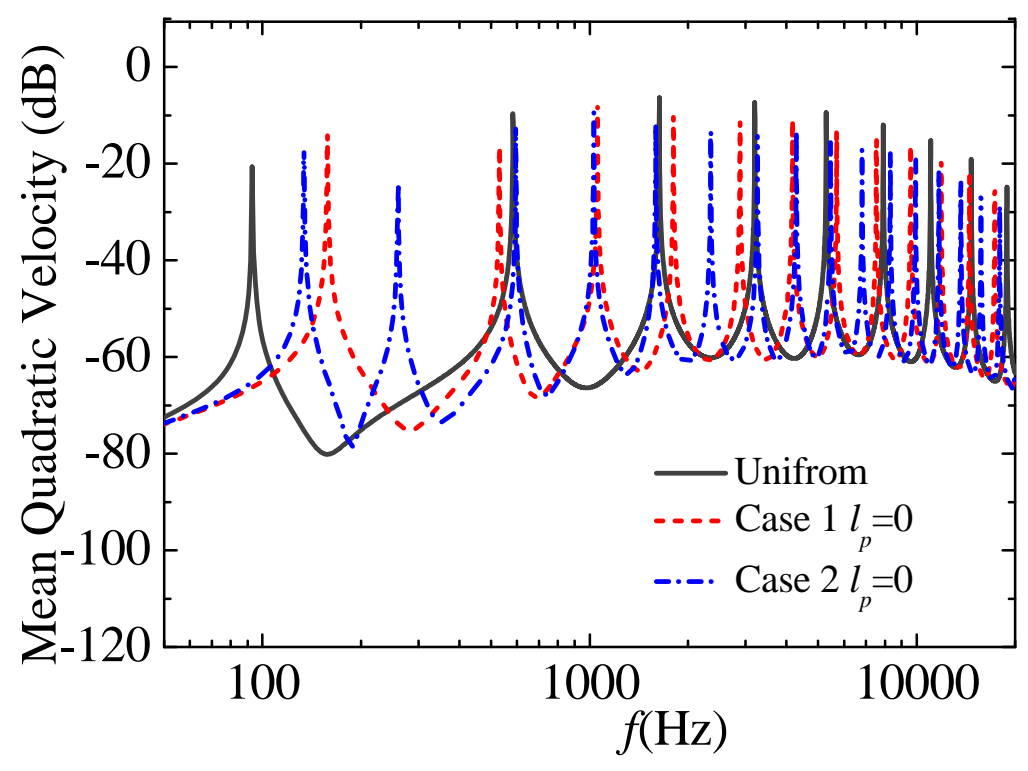

(b)

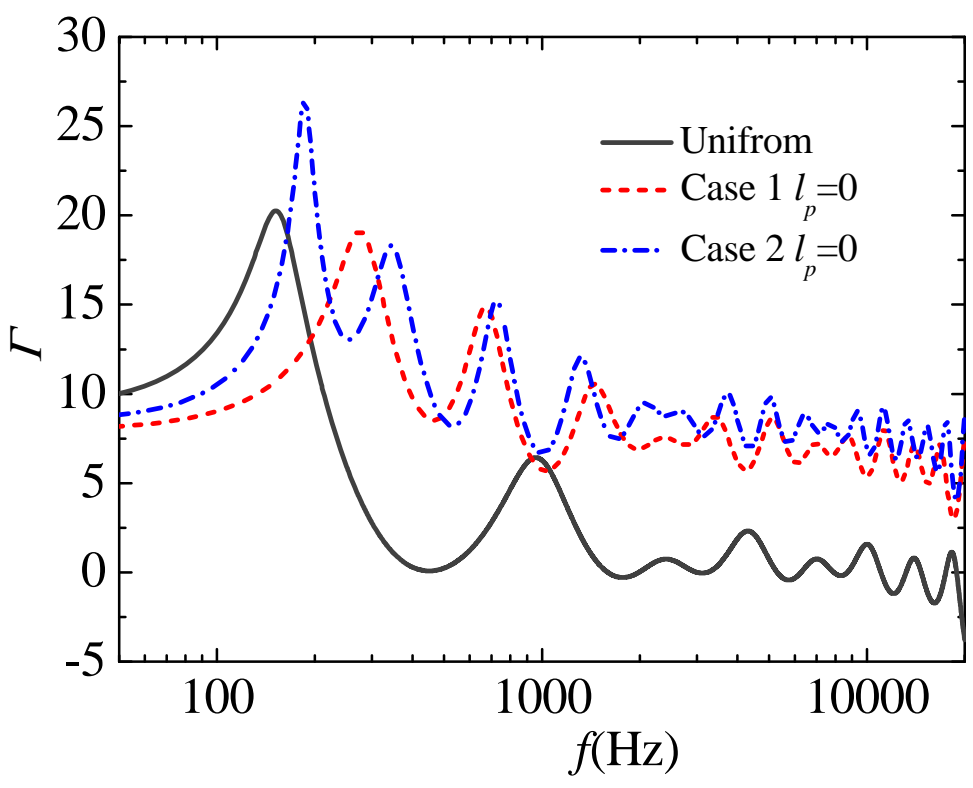


Fig. 3

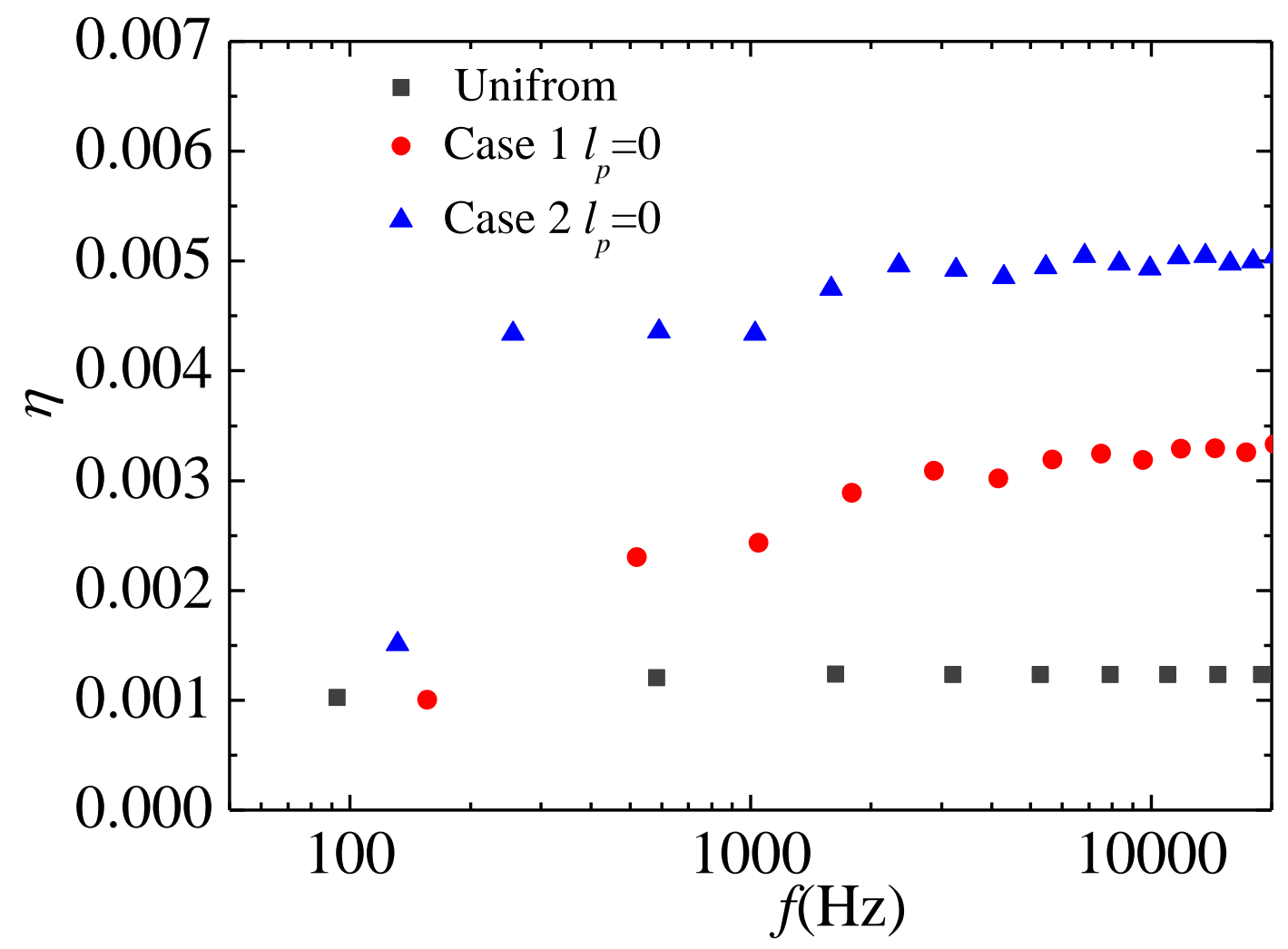


Fig. 4

(a)

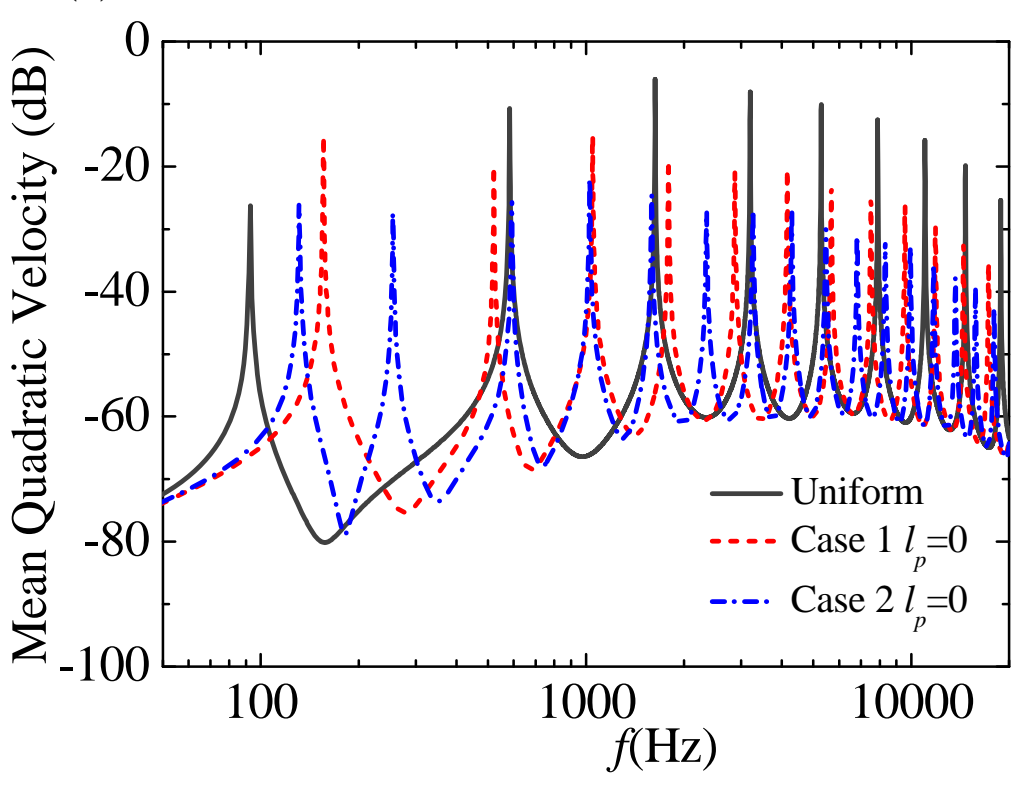

(b)

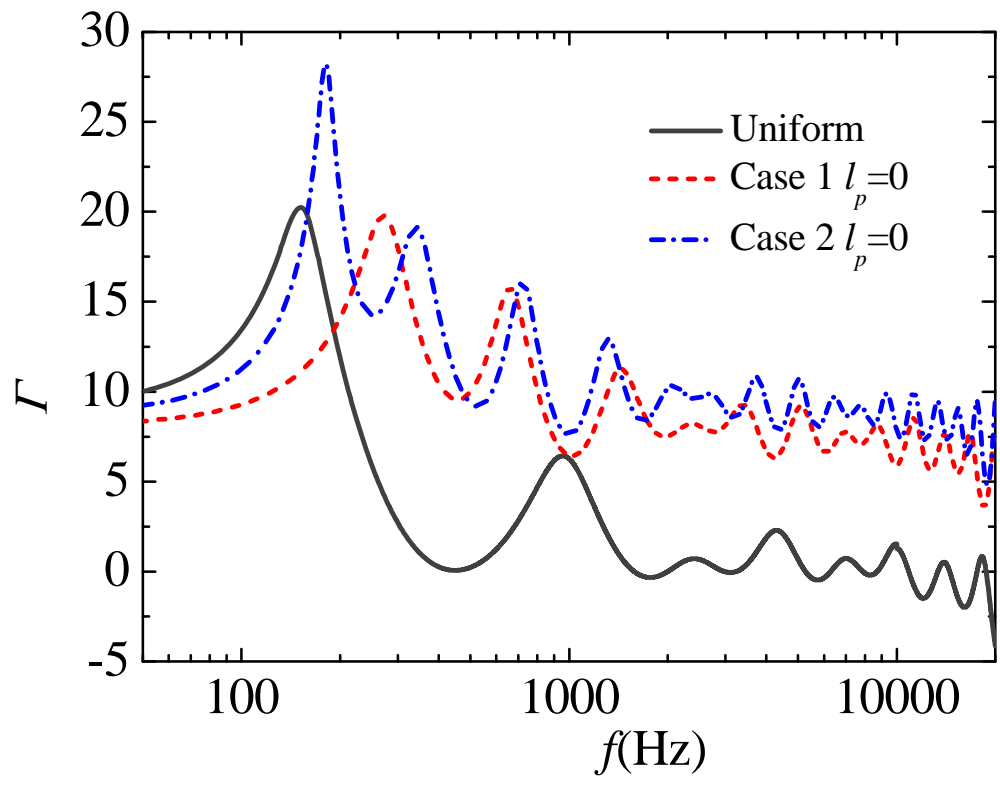


Fig. 5

(a)

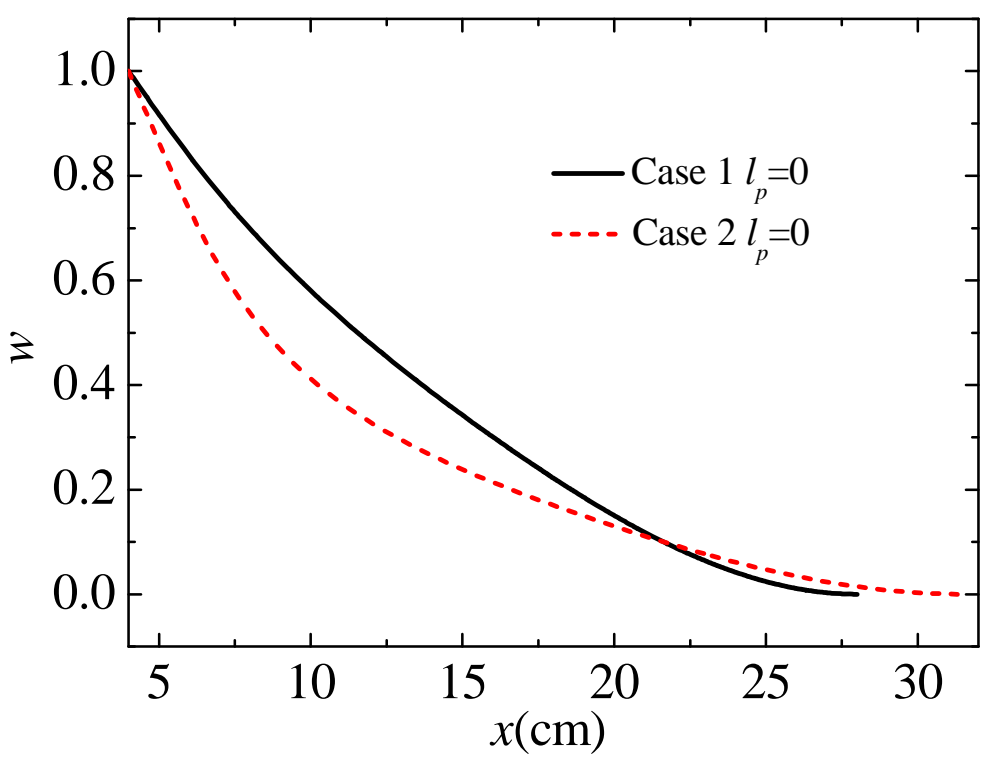

(b)

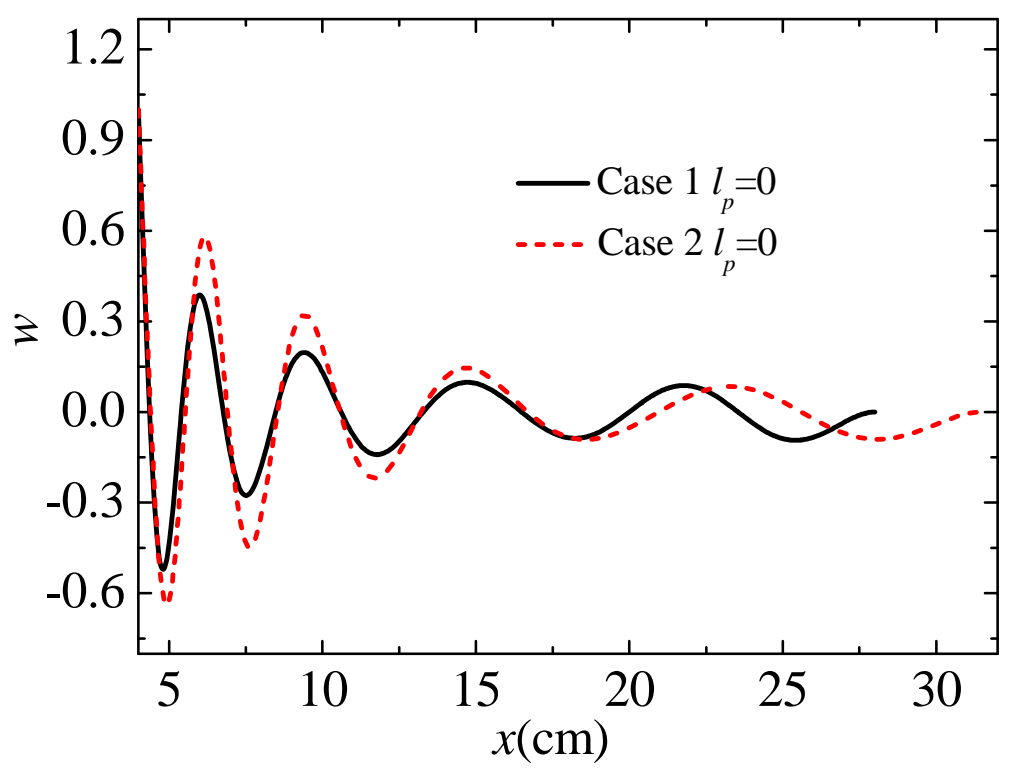


Fig. 6

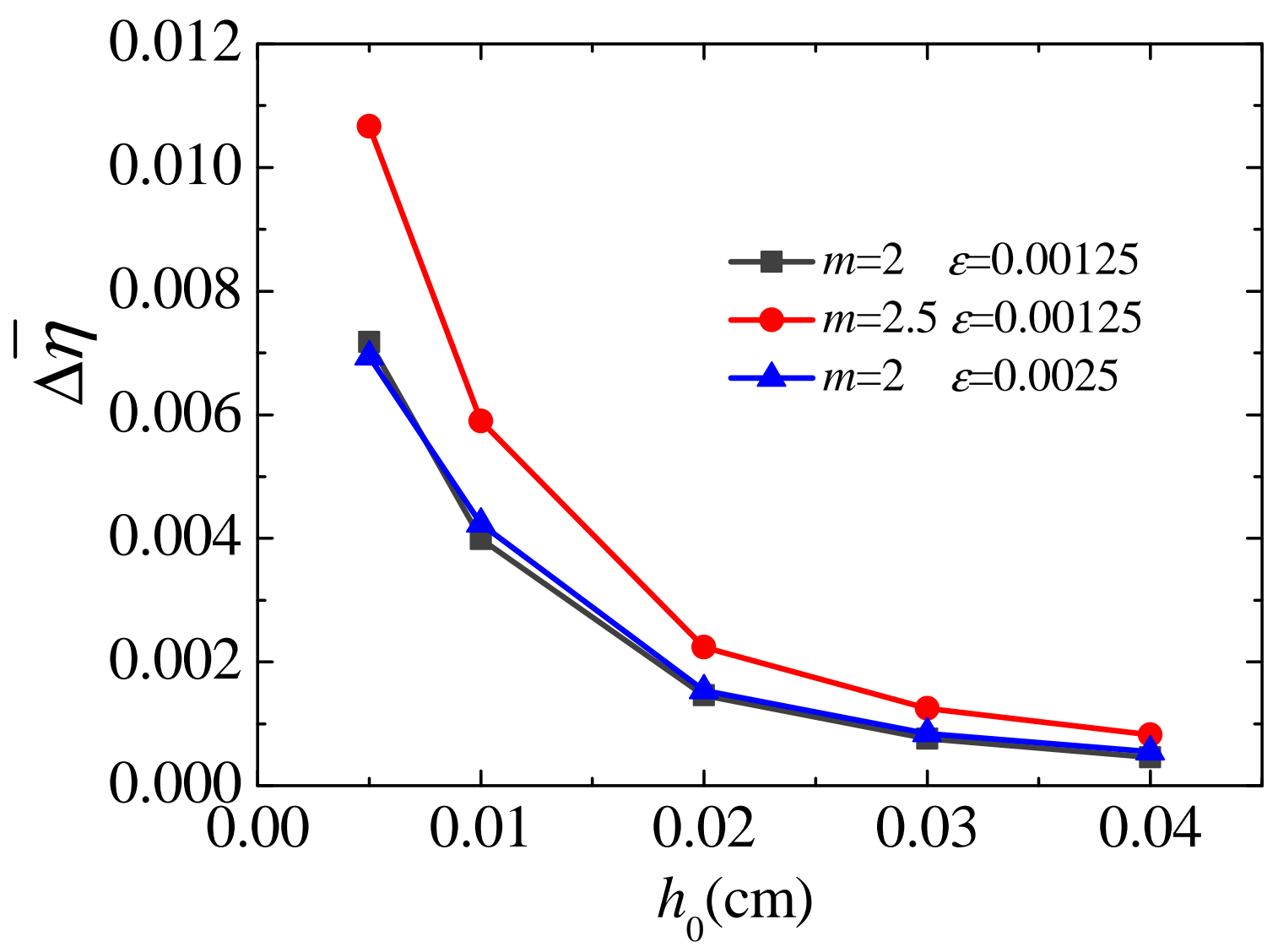


Fig. 7

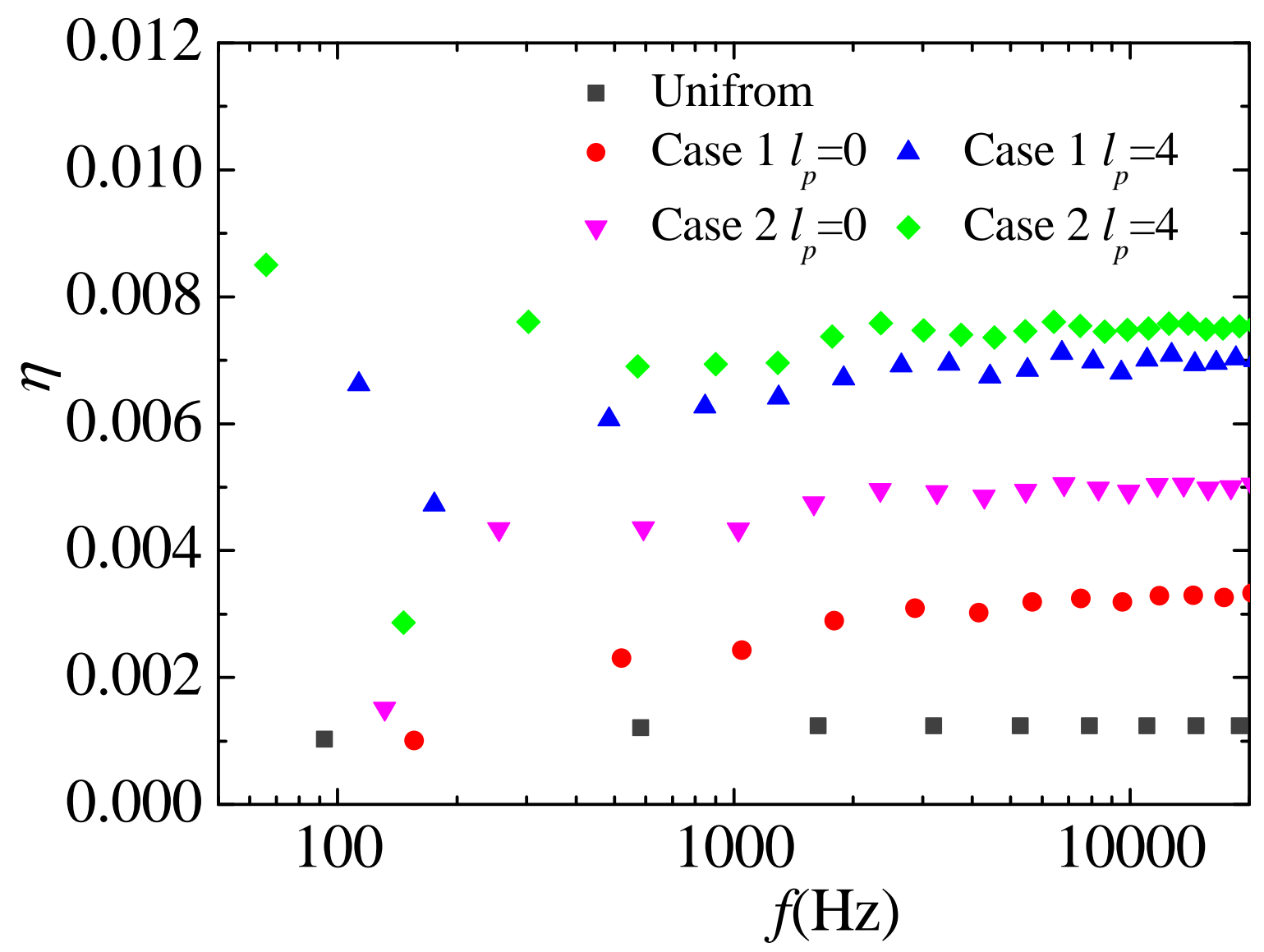


Fig. 8

(a)

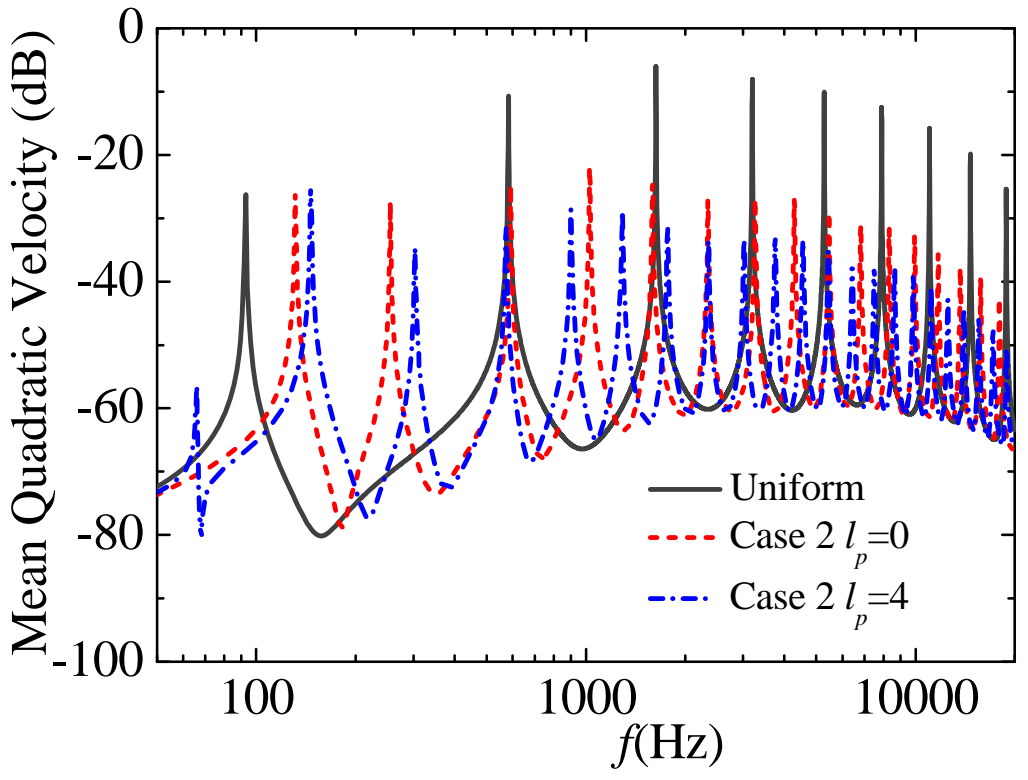

(b)

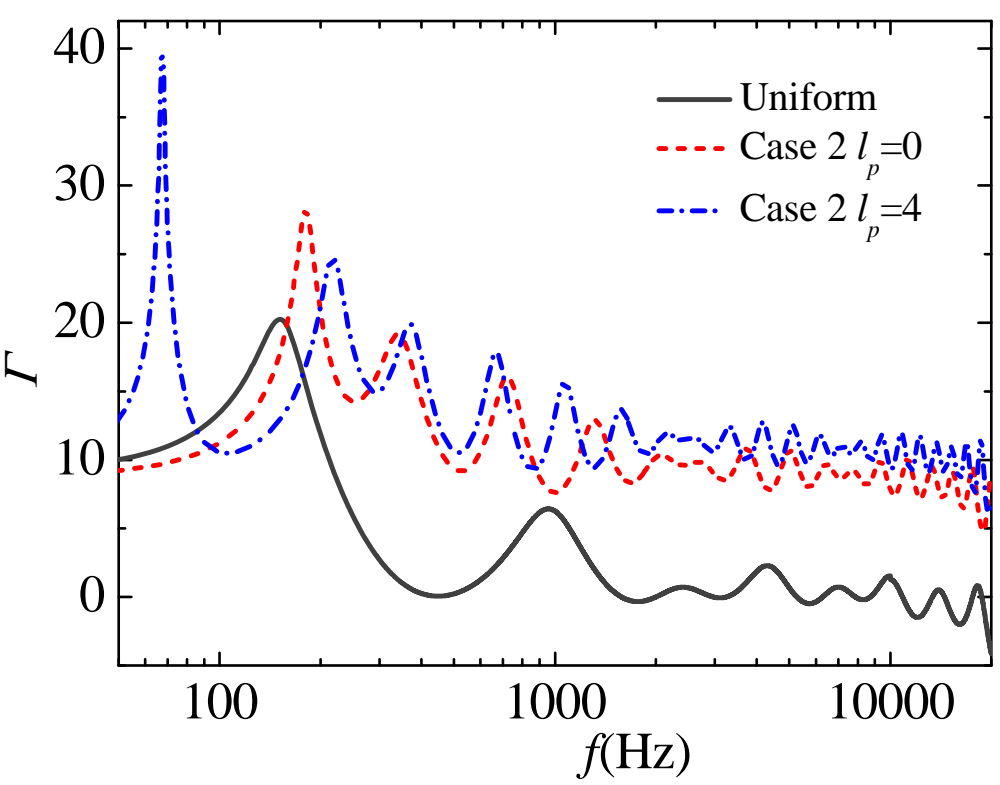


Fig. 9

(a)

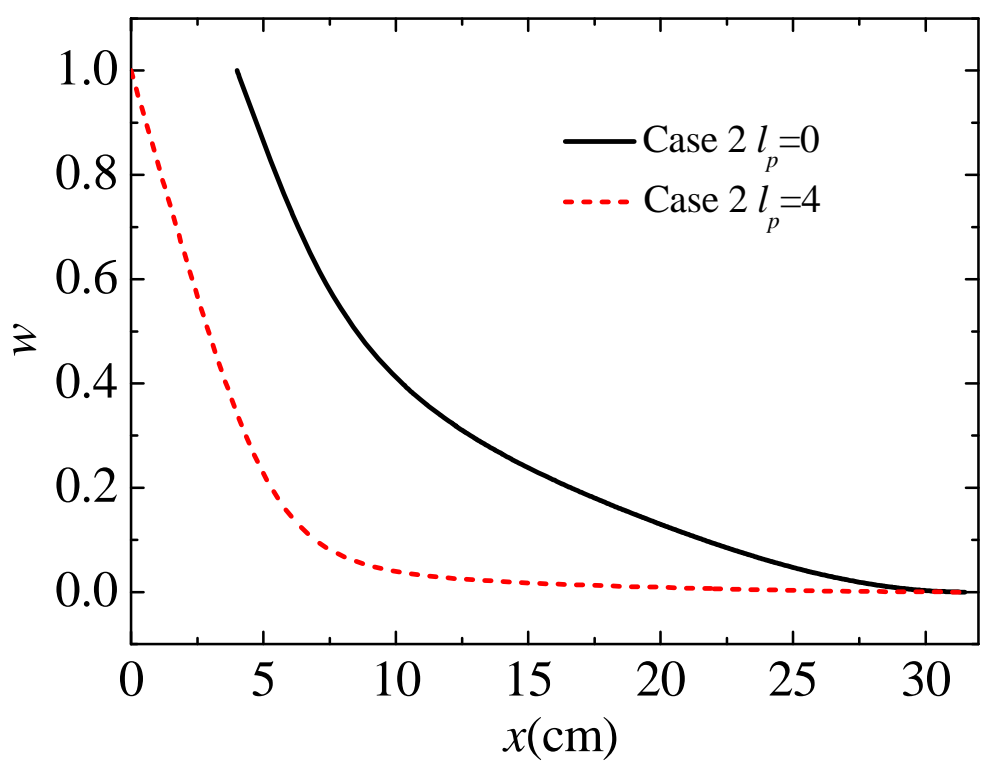

(b)

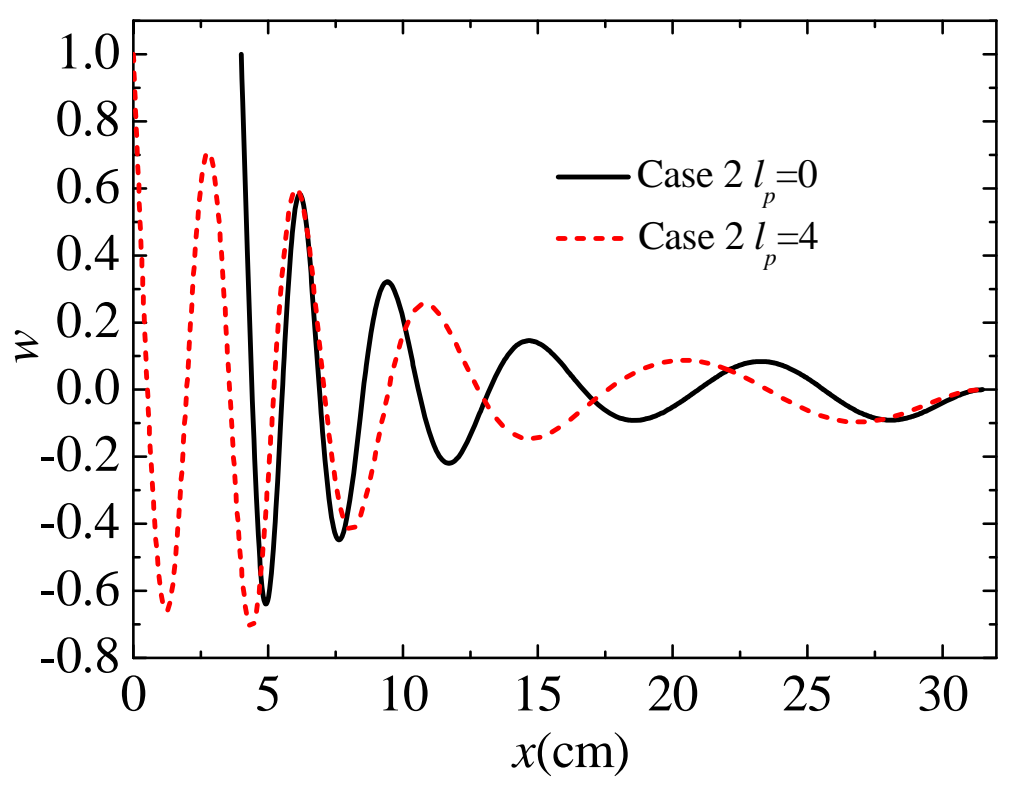


Fig 10

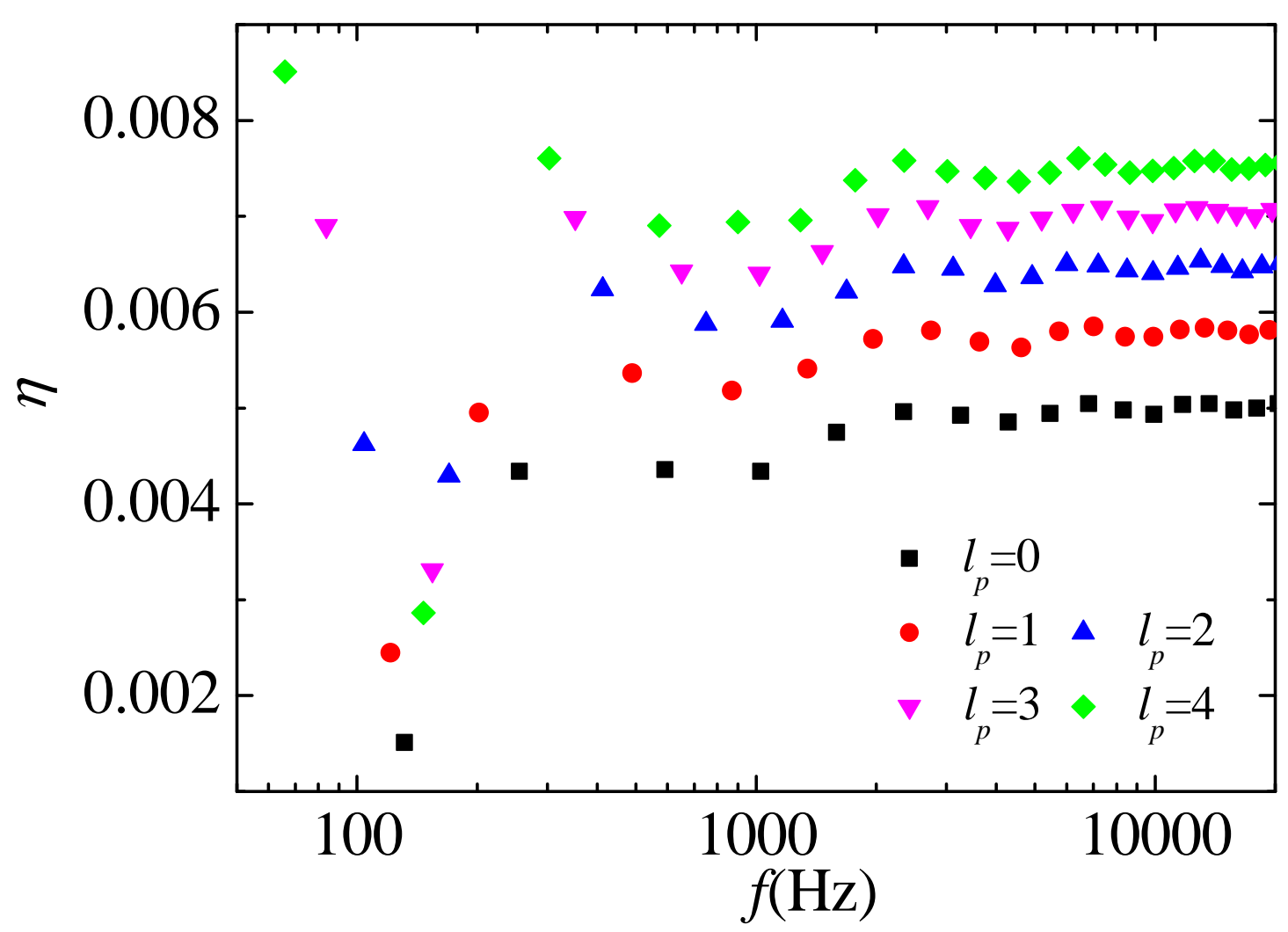


Fig. 12
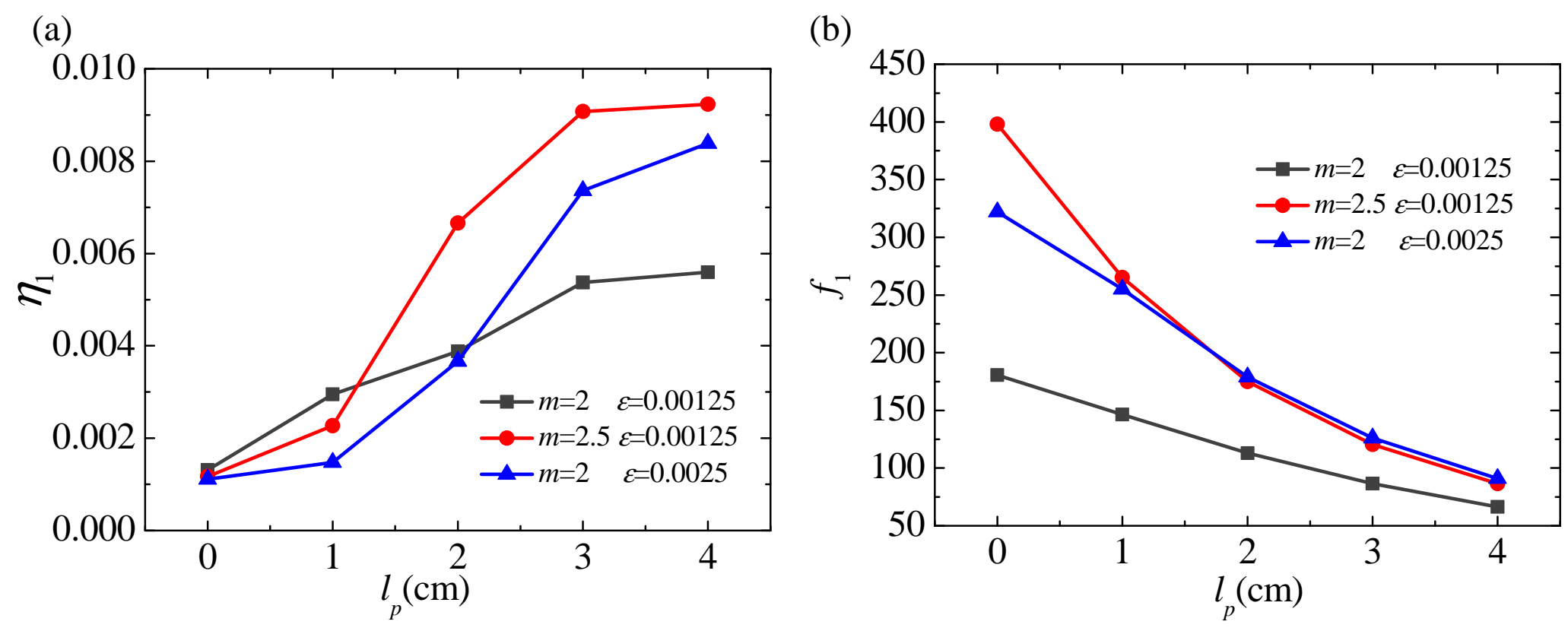